\title{
Sensitivity optimization of a rhodopsin-based fluorescent voltage indicator
}

Ahmed S Abdelfattah 1,2,3*\#, Jihong Zheng ${ }^{3 *}$, Daniel Reep ${ }^{3,4}$, Getahun Tsegaye ${ }^{3,4}$, Arthur Tsang ${ }^{3,4}$, Benjamin J Arthur ${ }^{3}$, Monika Rehorova ${ }^{5}$, Carl VL Olson ${ }^{5}$, Yi-Chieh Huang ${ }^{6}$, Yichun Shuai ${ }^{3}$, Minoru Koyama ${ }^{3}$, Maria V Moya ${ }^{7}$, Timothy D Weber $^{7}$, Andrew L Lemire ${ }^{3}$, Christopher A Baker ${ }^{8,9}$, Natalie Falco ${ }^{3}$, Qinsi Zheng ${ }^{3}$, Jonathan B Grimm ${ }^{3}$, Mighten C Yip ${ }^{10}$, Deepika Walpita ${ }^{3}$, Craig R Forest ${ }^{10}$, Martin Chase ${ }^{11}$, Luke Campagnola ${ }^{8}$, Gabe Murphy ${ }^{8}$, Allan M Wong ${ }^{3,4}$, Jerome Mertz ${ }^{7}$, Michael N Economo ${ }^{7}$, Glenn Turner ${ }^{3,4}$, Bei-Jung Lin ${ }^{6}$, Tsai-Wen Chen ${ }^{6}$, Ondrej Novak ${ }^{5}$, Luke D Lavis ${ }^{3}$, Karel Svoboda ${ }^{3,4}$, Wyatt Korff3,4, Eric R Schreiter ${ }^{3,4+\#, ~ J e r e m y ~ P ~ H a s s e m a n ~}{ }^{3,4+\#, ~ I l y a ~ K o l b 3,4 † \# ~}$

${ }^{1}$ Department of Neuroscience, Brown University, Providence, RI 02906, USA

${ }^{2}$ Carney Institute for Brain Science, Brown University, Providence, RI 02906, USA

3 Janelia Research Campus, Howard Hughes Medical Institute, Ashburn, VA 20147, USA

${ }^{4}$ GENIE Project Team

${ }^{5}$ Department of Physiology, Second Faculty of Medicine, Charles University, Plzenska 130/221, CZ-15006 Prague 5, Czech Republic

${ }^{6}$ Institute of Neuroscience, National Yang Ming Chiao Tung University, Taipei, 112, Taiwan

${ }^{7}$ Department of Biomedical Engineering, Boston University, 44 Cummington Mall, Boston MA 02215, USA

${ }^{8}$ Allen Institute, Seattle, WA, USA

${ }^{9}$ Department of Neurology, Yale School of Medicine, New Haven, CT, USA

${ }^{10}$ George W. Woodruff School of Mechanical Engineering, Georgia Institute of Technology, 315 Ferst Dr NW Atlanta, GA, USA, 30332

${ }^{11}$ Unaffiliated

${ }^{*}$ contributed equally to this work

${ }^{\dagger}$ co-supervised this work

${ }^{\#}$ Corresponding authors:

- ASA: ahmed abdelfattah@brown.edu

- ERS: schreitere@janelia.hhmi.org

- JPH: hassemani@janelia.hhmi.org

- IK: kolbi@janelia.hhmi.org

\section{Abstract}

The ability to optically image cellular transmembrane voltage at millisecond-timescale resolution can offer unprecedented insight into the function of living brains in behaving animals. The chemigenetic voltage indicator Voltron is bright and photostable, making it a favorable choice for long in vivo imaging of neuronal populations at cellular resolution. Improving the voltage sensitivity of Voltron would allow better detection of spiking and subthreshold voltage signals. We performed site saturation mutagenesis at 40 positions in Voltron and screened for increased $\Delta F / F_{0}$ in response to action potentials (APs) in neurons. Using a fully automated patchclamp system, we discovered a Voltron variant (Voltron.A122D) that increased the sensitivity to a single AP by $65 \%$ compared to Voltron. This variant (named Voltron2) also exhibited approximately 3-fold higher sensitivity in response to sub-threshold membrane potential changes. Voltron2 retained the sub-millisecond kinetics and photostability of its predecessor, with lower baseline fluorescence. Introducing the same A122D substitution to other Ace2 opsin-based voltage sensors similarly increased their sensitivity. We show that Voltron2 enables improved sensitivity voltage imaging in mice, zebrafish and fruit flies. Overall, we have discovered a generalizable mutation that significantly increases the sensitivity of Ace2 rhodopsin-based sensors, improving their voltage reporting capability. 


\section{Introduction}

Genetically encoded voltage indicators (GEVIs) have served as an enabling technology for visualizing neuronal activity at unprecedented spatiotemporal resolution(Hochbaum et al. 2014; Lin and Schnitzer 2016; Xu et al. 2017). Nevertheless, optical imaging of voltage using GEVIs presents many challenges for the design of these proteins. An ideal voltage sensor must concurrently fulfill many requirements, including but not limited to: (1) high sensitivity to membrane potential changes of a neuron, (2) fluorescence changes that are fast enough to follow and accurately report APs and (3) high degree of localization to neuron outer membranes. Further requirements may be desirable depending on application, such as sensitivity to sub-threshold membrane potential changes, photostability, and compatibility with two-photon excitation.

One approach to engineering GEVIs involves exploiting the native voltage sensitivity of microbial rhodopsins. The opsin Archaerhodopsin 3 (Arch) was first successfully used to optically record APs in neuronal culture (Kralj et al. 2012); however, it was found to be too dim at physiologically tolerable imaging powers for in vivo applications. Subsequent protein engineering efforts of Arch yielded improvements in brightness as well as sensitivity, kinetics, and reduced photocurrents (Chien et al. 2021; Flytzanis et al. 2014; Gong et al. 2013; Hochbaum et al. 2014; Mclsaac et al. 2014; Piatkevich et al. 2018). An alternative strategy to develop bright rhodopsin-based GEVIs is to create a Förster resonance energy transfer (FRET) pair between a bright fluorescent protein (FP) and the rhodopsin protein (Gong et al. 2015; Zou et al. 2014). In this strategy, the bright FP is the reporter fluorophore, and the rhodopsin is used as the voltage sensitive domain. This strategy was successfully implemented to develop Ace2N-mNeon, a bright fast GEVI that was able to report single APs in vivo (Gong et al. 2015).

The Ace2N-mNeon member of the rhodopsin family of GEVIs has been used as a scaffold to create GEVIs with other favorable characteristics. A red GEVI called VARNAM consisting of Ace2N fused to a red FP mRuby3 displayed high sensitivity, good in vivo performance, and a spectral shift that made it compatible with blue-shifted optogenetic probes (Kannan et al. 2018). Our group has previously replaced the FP in Ace2N$\mathrm{mNeon}$ with a HaloTag protein (Los et al. 2008) covalently bound to a small-molecule fluorophore (JaneliaFluor or JF (Grimm et al. 2015, 2017)) to create a chemigenetic sensor called Voltron (Abdelfattah et al. 2019). The introduction of three point mutations to the rhodopsin domain of Voltron led to Positron, a positive-going GEVI with sensitivity and kinetics comparable to the original Voltron (Abdelfattah et al. 2020).

Encouraged by the ability of point mutations in the rhodopsin domain to alter function, we performed a largescale screen of point mutations to find improved versions of Voltron. We discovered that the introduction of an A122D mutation increased the sensitivity of Voltron, particularly in the sub-threshold range, without compromising kinetics, membrane trafficking or photobleaching. Thus Voltron.A122D was named Voltron2 as a next-generation version of the sensor. Consistent with the observation in culture, in vivo imaging in flies, zebrafish and mice revealed an increased signal-to-noise ratio (SNR) of Voltron2 compared to Voltron. 


\section{Methods}

\section{$\underline{\text { Reagent availability }}$}

The following plasmids used in this study are available on Addgene:

- pAAV-syn-FLEX-Ace2N-4AA-mNeon-ST A122D WPRE (\#172908)

- $\quad$ pGP-pcDNA3.1 Puro-CAG-Voltron2 (\#172909)

- $\quad$ pGP-CAG-Ace2N-4AA-mNeon A122D-WPRE-bGH-polyA (\#172911)

- pGP-CAG-Ace2N-4AA-mNeon-ST A122D-WPRE-bGH-polyA (\#172912)

The $\mathrm{JF}_{549}$-HaloTag ligand is available from Promega; all other dyes can be requested at dyes.janelia.org.

\section{Single-site directed mutagenesis}

The cloning vector pcDNA3.1/Puro-CAG- Ace2N_HaloTag expression vector (Invitrogen) was modified by moving the $\mathrm{Kpnl}$ site from outside of the insert to the junction between Ace2N domain and the Halo-tag. The subsequent vector was digested by Nhel/Kpnl cleaving out the Ace2N domain. End PCR primers were designed 30bp upstream of Nhel site (5'-GCTCACAAATACCACT-3') and 38 bp downstream of new Kpnl site (5'-CCAGGACTTCCACATAA-3'). Overlapping internal primers were designed for each of 40 targeted amino acid residues in the Ace2N domain. One primer of the pair contained the degenerate codon NNS and the other primer a 27-30bp complementary overhang. When paired with the end primers two amplicons were created (Phusion High-Fidelity DNA Polymerase; NEB) that overlap with each other and the digested vector ends. Each set of overlapping paired amplicons (37.5 fmol each) were assembled with the digested pcDNA3.1/PuroCAG backbone (25 fmol) using an isothermal assembly reaction (Gibson et al. 2009). Each $20 \mathrm{uL}$ reaction mix consisted of $5 \mathrm{X}$ isothermal assembly buffer (25\% PEG-8000, $500 \mathrm{mM}$ Tris- $\mathrm{HCl}$ pH 7.5, 50mM MgCl2, 50mM DTT, $1 \mathrm{mM}$ each dNTP and 5mM NAD), T5 exonuclease (0.08 U, NEB), Taq DNA Ligase (80 U, NEB), Phusion HF DNA Polymerase (0.5 U, NEB). The reactions were incubated at $500 \mathrm{C}$ for 30-60 minutes. Reactions were transformed into STABL2 chemically competent E. coli cells (ThermoFisher) and plated on LB/Amp agar plates and incubated at $370 \mathrm{C}$ for $16-20$ hours.

For each site library, 96 colonies were picked into $2.6 \mathrm{~mL}$ of $2 \mathrm{x}-\mathrm{YT}$ media $(2 \times 1.3 \mathrm{~mL}$ in $2 \mathrm{~mL}$ deep-well culture plates) and grown for 24 hours, 225 rpm @37C with Ampicillin (100mg/L). The cultures were pelleted at 3200 $\mathrm{x} \mathrm{g}$ and frozen at -80C. For each plate plasmids were extracted using the E-Z 96 FastFilter Kit (Omega BioTek) and eluted into a half-area UV transparent 96 well plate (Corning Costar). Each of the plasmid plates was concentration normalized to $60 \mathrm{ng} / \mathrm{ul}$ by reading the $260 \mathrm{~nm}$ absorbance (Tecan Infinite M1000Pro) followed by custom dilution (Hamilton Nimbus). Variant plasmids were arrayed along with controls for highthroughput electroporation of neuronal cell culture (Hamilton STAR). Top performing variants from the subsequent neuronal culture screen were Sanger-sequenced to determine their mutation as well as the entire library being sequenced using a next-generation deep-sequencing approach (Supplementary Methods). 


\section{Combinatorial mutagenesis}

Top-performing single-site mutations (Y63L, N69E, V74EN, R78H, N81S, L89A/C/G/T, A122D/H, V196P) were recombined to test all possible combinations (1423). All combinations could be recapitulated using two overlapping amplicons covering the Ace2N domain. Some mutations (Y63L, A122D/H, V196P) were introduced as part of the PCR template and others (N69E, V74E/W, R78H, N81S, L89A/C/G/T) by PCR primer. For the $\mathrm{N}$-term amplicon (305bp) twenty-four reverse primers were designed based on the wild-type anti-sense sequence (5'-

AGTGGTGTGGTCAGCACCCAGTTAATATATCTTGCGTAGACCACCTGCCTTTCACCATTCATTGTCAGGTC C-3') and included every combination of N69E, V74E/W, R78H, N81S. Forty-eight unique N-term amplicons were created by combining these twenty-four reverse mutagenic primers, the upstream end primer (5'GCTCACAAATACCACT-3') and templates with and without Y63L. For the C-term amplicon (493bp) 10 forward primers were designed based on the wild-type sense sequence (5'-

ATATTAACTGGGTGCTGACCACACCACTGCTCCTGCTCGATCTCATCGTCATGACCAAGATGGGCGGAGT GA -3') and included every combination of N81S, L89A/C/G/T. Sixty unique C-term amplicons were created by combining 10 forward mutagenic primers, the downstream end primer (5'- CCAGGACTTCCACATAA-3') and templates each containing a combination of $\mathrm{A} 122 \mathrm{D} / \mathrm{H}$ and $\mathrm{V} 196 \mathrm{P}$. The $\mathrm{N}$-term and $\mathrm{C}$-term amplicon libraries overlapped by 28 bp (5'-ATATTAACTGGGTGCTGACCACACCACT-3') and included the N81 site in both. The PCR products were gel extracted, quantified and normalized to $18.75 \mathrm{fmol} / \mathrm{ul}$. The Nhel/Kpnl digested pcDNA3.1/Puro-CAG backbone was normalized to $12.5 \mathrm{fmol} / \mathrm{ul}$. Using a liquid-handling robot (Hamilton STAR) the N-term and C-term amplicons sets were pairwise combined (2uL each) along with the Nhel/Kpnl digested pcDNA3.1/Puro-CAG vector (2uL) to create 1423 unique isothermal assembly reactions in 96 well thermocyler plates. The plates were reacted and transformed as above in 96 well plates. Approx. 35uL of each transformant was robotically dispensed into the corresponding wells of two 48 well Q-trays (Genetix) containing LB/Amp agar. Q-trays were incubated for $16-20$ hours at $37^{\circ} \mathrm{C}$ and two colonies were picked from each well and separately cultured, pelleted and frozen in 96 well deep well plates. Plasmids were extracted from the 96 well pellets and concentration normalized as above. Once verified by Sanger sequencing the combinatorial variants were arrayed for electroporation of neuronal cell culture and the subsequent field stimulation assay.

\section{Neuronal cell culture}

Experiments were conducted in accordance with guidelines for animal research approved by the Janelia Research Campus Institutional Animal Care and Use Committee. Neonatal rat pups (Charles River Laboratory) were euthanized and neocortices (for field stimulation experiments) or hippocampi (for patch-clamp experiments), were isolated. Tissue was dissociated using papain (Worthington) in $10 \mathrm{mM} \mathrm{HEPES} \mathrm{pH} 7.4$ in Hanks' Balanced Salt Solution for $30 \mathrm{~min}$ at $37^{\circ} \mathrm{C}$. Suspensions were triturated with a Pasteur pipette and passed through a $40-\mu \mathrm{m}$ strainer. Cells were transfected by combining $5 \times 10^{5}$ viable cells with $400 \mathrm{ng}$ plasmid DNA and nucleofection solution in a $25-\mu \mathrm{L}$ electroporation cuvette (Lonza). Cells were electroporated according to the manufacturer's protocol.

For the field stimulation screen, neurons were plated onto poly-D-lysine (PDL) coated, 96-well, glass bottom (\#1.5 cover glass) plates (MatTek) at $\sim 1 \times 10^{5}$ cells per well in $100 \mu \mathrm{L}$ of a 4:1 mixture of NbActiv4 (BrainBits) and plating medium (28 mM glucose, $2.4 \mathrm{mM} \mathrm{NaHCO}, 100 \mu \mathrm{g} / \mathrm{mL}$ transferrin, $25 \mu \mathrm{g} / \mathrm{mL}$ insulin, $2 \mathrm{mM} \mathrm{L-}$ 
glutamine, $100 \mathrm{U} / \mathrm{mL}$ penicillin, $10 \mu \mathrm{g} / \mathrm{mL}$ streptomycin, 10\% FBS in MEM). The next day, $190 \mu \mathrm{L}$ of NbActiv4 medium was added to each well. Plates were incubated at $37^{\circ} \mathrm{C}$ and $5 \% \mathrm{CO} 2$, to be imaged after $12-15$ days in culture. Typically, 8 wells of a 96-well plate were electroporated with Voltron (as a control) and the remaining wells were electroporated with constructs of interest ( 4 wells per construct). The first and last columns of the plate were not used.

For patch-clamp, $\sim 2 \times 10^{5}$ cells were plated onto PDL-coated, 35-mm glass bottom plates (Mattek, \#0 cover glass) in $120 \mu \mathrm{L}$ of a 1:1 mixture of NbActiv4 and plating medium in the center of the plate. The next day, $2 \mathrm{~mL}$ of NbActiv4 medium was added to each plate. Plates were incubated for 7-13 days prior to beginning experiments.

\section{Field stimulation assay in neuronal culture}

To prepare the neurons for field stimulation, they were first incubated for 1 hour in NbActiv4 media supplemented with $2 \mathrm{nM} \mathrm{JF}_{525}-\mathrm{HaloTag}_{\text {at }} 37^{\circ} \mathrm{C}$. They were then rinsed three times with imaging buffer containing (in mM) $140 \mathrm{NaCl}, 0.2 \mathrm{KCl}, 10$ HEPES, 30 glucose (pH 7.3-7.4) and left in a solution containing imaging buffer with added receptor blockers (10 $\mu \mathrm{M}$ CNQX, $10 \mu \mathrm{M}$ (R)-CPP, $10 \mu \mathrm{M}$ gabazine, $1 \mathrm{mM}(\mathrm{S})$ MCPG, Tocris) to reduce spontaneous activity (Wardill et al. 2013).

The field stimulation assay for GEVIs was adapted from our existing screening pipeline (Dana et al. 2016, 2019). Fluorescence was excited with a white LED (Cairn Research) through a custom filter cube (Excitation: 512/25 nm, Emission: 555/20 nm, dichroic: $525 \mathrm{~nm}$, Chroma) and imaged using a 40X/0.6 NA objective (Olympus) with an EMCCD camera (Ixon Ultra DU897, Andor). To enable high-speed imaging, an Optomask (Cairn Research) was used to mask out camera pixels outside a 256x256 center square. Reference images of each field of view (FOV) were taken at full sensor frame, $100 \mathrm{~ms}$ exposure. For high-speed imaging during stimulation, we applied $8 x$ binning, $0.01 \mathrm{~ms}$ exposure, and $25 \mathrm{EM}$ gain for a resulting frame rate of 1,497 $\mathrm{Hz}$.

For each well in the 96-well plate, either 9 FOVs surrounding the center of the well were chosen, or a machine vision function utilizing ilastik (Berg et al. 2019) was used to automatically focus on cell somata. For each FOV, first, a reference image was acquired, and then, neuronal APs were evoked by field stimulation ( 8 pulses, $40 \mathrm{~V}$, $1 \mathrm{~ms}, 8.3 \mathrm{~Hz}$; S-48, Grass Instruments) concurrently with high-speed imaging. The camera 'fire' signal and the stimulator sense line were used to determine the frame at which the stimulation occurred.

To correct for photobleaching, a single exponential with three free parameters was fit to the time series for each pixel. Frames succeeding each electrical stimulus (during which the response nominally occurred) were excluded from the fit. The value of the fitted bleach function at the first frame was taken as the baseline fluorescence for that pixel. Background fluorescence was computed as the $1^{\text {st }}$ percentile of the baseline fluorescence across all pixels.

Responses to the eight electrical pulses within each recording were averaged using the timings derived from the camera and electrode triggers. For each pixel, a Mann-Whitney $U$ test was performed between the frames preceding the average response $(20 \mathrm{~ms})$ and 10, 20, and $40 \mathrm{~ms}$ of frames succeeding it. Pixels with a $p$ value $<0.001$ for any of these three tests were considered responsive and averaged together to contribute to 
the $\Delta \mathrm{F} / \mathrm{F}_{0}$ trace. Traces were fit with the product of a rising and decaying exponential to capture both the on and off kinetics. The fit was used to calculate the characteristics of the variant such as maximum $\Delta F / F_{0}$ and kinetics (10-90\% rise and decay times).

Pixel statistics were pooled across all the wells in each plate that contained the construct of interest. Wells with fewer than four responsive pixels were considered to be unresponsive and discarded from analysis.

For every plate in the field stimulation assay, a percent detectable improvement (PDI) statistic was calculated to answer the question: "Given the variability of Voltron control wells in the plate, what is the minimum improvement in $\Delta F / F_{0}$ that can be reliably detected?". That is, a PDI of $20 \%$ for a plate indicates that a $\geq 20 \%$ improvement in $\Delta F / F_{0}$ over Voltron can be considered statistically meaningful. Large PDI values are undesirable because they indicate high variability in the control responses. PDI is calculated as follows: $100 *($ mean $(x)$-quantile $(x, 0.01)) /$ mean $(x)$, where $x$ is the $\Delta F / F_{0}$ of Voltron control well pixels, sampled 10,000 times with replacement. The PDI was one of the parameters used to screen out poorly responding variants.

Normalization to in-plate Voltron controls was useful to reduce the effects of within-plate and within-week variability. Pixels from each variant were pooled across wells. For each variant, the median was taken from this pool and divided by the median from the control pool to perform the normalization. Significance values for each variant were determined using a Mann-Whitney $U$ test between the pools.

\section{Automated whole-cell electrophysiology}

Cultured neurons were patch-clamped at 7-13 DIV at room temperature $\left(23^{\circ} \mathrm{C}\right)$. On the day of the experiment, cell culture medium was first rinsed with imaging buffer consisting of (in mM): $145 \mathrm{NaCl}, 2.5 \mathrm{KCl}, 10 \mathrm{D}$ -

Glucose, 10 HEPES, $2 \mathrm{CaCl}$, $1 \mathrm{MgCl}$ ( $\mathrm{pH} 7.3$, adjusted to $310 \mathrm{mOsm}$ with sucrose). The cells were then incubated with $100 \mathrm{nM} \mathrm{JF}_{525}$ dye for 10 minutes (for Voltron mutant screening only), rinsed twice, and kept in imaging buffer. For voltage clamp recordings, $1 \mu \mathrm{M}$ TTX was added to the bath to suppress the generation of APs. Micropipettes were pulled on a horizontal puller (P-97, Sutter Instruments) to a tip resistance of 3 to 6 $\mathrm{M} \Omega$. For voltage clamp experiments, pipettes were filled with cesium-based internal solution containing (in mM): 115 CsMeSO4, 15 CsCl, 3.5 Mg-ATP, 5 NaF, 10 EGTA, 10 HEPES, 3 QX-314 (pH 7.3-7.4, 280-290 mOsm). For current clamp experiments, pipettes were filled with $130 \mathrm{KMeSO} 4,10 \mathrm{HEPES}, 5 \mathrm{NaCl}, 1 \mathrm{MgCl} 2,1$ Mg-ATP, 0.4 Na-GTP, 14 Tris-phosphocreatine (pH 7.3-7.4, 280-290 mOsm).

To perform automated patch-clamp screening of the top-performing hits from the field stimulation screen, we used a custom-built Automated uM Workstation, manufactured by Sensapex (Oulu, Finland), based on the PatcherBot (Kolb et al. 2019). The system is built around an AxioObserver 7 inverted microscope (Zeiss), outfitted with a computer-controlled stage, micromanipulators, and pipette pressure controllers. Pipettes were automatically cleaned between every patch-clamp attempt with Tergazyme and reused, enabling higher throughput than possible with manual patch-clamp (Kolb et al. 2016, 2019). Electrophysiology recordings were performed with a Multiclamp 700B amplifier (Molecular Devices), and digitized with a multifunction data acquisition board (National Instruments PCle-6259). Neurons were imaged using a 40x/1.3 NA oil immersion objective (Zeiss), illuminated with high-power LEDs (Spectra-X light engine, Lumencor) and imaged with a 
digital sCMOS camera (Hamamatsu Orca Flash 4.0). To image Voltron 525 , we used a filter cube containing 510/25 nm excitation filter, 545/40 emission filter, $525 \mathrm{~nm}$ dichroic (Chroma), with a measured power of 14.7 $\mathrm{mW} / \mathrm{mm}^{2}$ in the imaging plane. To image Ace2N-mNeon, the filter cube contained a 470/24 nm excitation filter, $525 / 40 \mathrm{~nm}$ emission filter, $506 \mathrm{~nm}$ dichroic with a measured power of $18.1 \mathrm{~mW} / \mathrm{mm}^{2}$ in the imaging plane. To image VARNAM, the filter cube contained $575 / 25 \mathrm{~nm}$ excitation filter, 610LP emission filter, $594 \mathrm{~nm}$ dichroic, with a measured power of $32.8 \mathrm{~mW} / \mathrm{mm}^{2}$.

The uM Workstation was controlled by the Python platform Acq4 (Campagnola et al. 2014), modified to perform fully automated electrophysiology (www.acq4.org). To generate fluorescence/voltage curves, the membrane potential was stepped from +50 to $-110 \mathrm{mV}$ in $20 \mathrm{mV}$ increments from a resting potential of $-70 \mathrm{mV}$ ( $0.5 \mathrm{~s}$ baseline, $1 \mathrm{~s}$ step). For current clamp recordings, a short current pulse was injected ( $2 \mathrm{nA}, 2 \mathrm{~ms})$ to evoke APs.

Stimulus timing, baseline fluorescence calculation, background subtraction, and photobleaching correction was performed the same way as for the field stimulation assay. To identify responsive pixels, a Mann-Whitney $U$ test was performed between the baseline and voltage step segments of the recording. The $\mathrm{P}$ value criterion to identify responsive pixels was empirically set to $1 \mathrm{e}-10$.

The onset of each step was fit with the product of a rising and decaying exponential to capture the transient response (if any), summed with a single rising exponential to capture the steady-state response. The decay response was fit with a single exponential. Peak $\Delta F / F_{0}$ as well as onset and decay kinetics were calculated at each voltage step as was done for field stimulation.

\section{Imaging and whole-cell recording in brain slices}

All animal work was performed according to Institutional Animal Care and Use Committee approved protocols. Stereotaxic injections were made into right visual cortex ( $3.8 \mathrm{~mm}$ posterior and $3.0 \mathrm{~mm}$ lateral from bregma) of 4-week-old Sst-IRES-Cre driver mice under isofluorane anesthesia. Two injections of $200 \mathrm{~nL}$ each of AAV2/1syn-Flex-Voltron ${ }_{585}$-ST and AAV2/1-syn-Flex-Voltron2 ${ }_{585}$-ST and were targeted to 300 and $600 \mu \mathrm{m}$ below the cortical surface.

Four weeks later, isoflurane anesthetized mice were transcardially perfused with ice-cold NMDG slicing solution containing (in mM): $98 \mathrm{HCl}, 96 \mathrm{~N}$-methyl-d-glucamine (NMDG), $2.5 \mathrm{KCl}, 25 \mathrm{D}$-Glucose, $25 \mathrm{NaHCO}$, 17.5 HEPES, $12 \mathrm{~N}$-acetylcysteine, $10 \mathrm{MgSO}$, $5 \mathrm{Na-L-Ascorbate,} 3$ Myo-inositol, 3 Na Pyruvate, 2 Thiourea, $1.25 \mathrm{NaH} 2 \mathrm{PO} 4 \cdot \mathrm{H} 2 \mathrm{O}, 0.5 \mathrm{CaCl} 2$, and 0.01 taurine. Acute $350 \mu \mathrm{m}$ parasagittal slices containing primary visual cortex from the right hemisphere were prepared with a Compresstome (Precisionary Instruments) in ice-cold NMDG slicing solution at a slice angle of $17^{\circ}$ relative to the sagittal plane. Slices were incubated for $10 \mathrm{~min}$ in NMDG slicing solution at $34^{\circ} \mathrm{C}$ and then transferred to artificial CSF (aCSF; in mM): $94 \mathrm{NaCl}, 25 \mathrm{D}-\mathrm{Glucose}, 25$ NaHCO3, 14 HEPES, $12.3 \mathrm{~N}$-acetylcysteine, $5 \mathrm{Na}$-L-Ascorbate, 3 Myo-inositol, $3 \mathrm{Na}$ Pyruvate, $2.5 \mathrm{KCl}, 2$ $\mathrm{CaCl}$, $2 \mathrm{MgSO} 4,2$ Thiourea, 1.25 NaH2PO4 H20, 0.01 Taurine. All solutions were maintained under constant carbogen $(95 \%$ O2; $5 \%$ CO2). 
To complete fluorescent labeling of Voltron-expressing cells, $1 \mathrm{nM}$ of $\mathrm{JF}_{585}$ was dissolved in $20 \mu \mathrm{l}$ dimethyl sulfoxide (DMSO) and $20 \mu \mathrm{L}$ of $20 \%$ Pluronic F-127 (w/w in DMSO). The solubilized dye was then added to 20 $\mathrm{mL}$ of oxygenated aCSF and incubated with the acute brain slices for $1 \mathrm{~h}$ at room temperature, after which the slices were removed to a holding chamber (BSK 12, Scientific Systems Design) containing $500 \mathrm{~mL}$ oxygenated aCSF without dye. Slices were kept in this latter solution for at least one hour at room temperature prior to any experiment.

Slices were visualized using oblique (Olympus; WI-OBCD) infrared illumination using 20× or 4× objectives (Olympus). Recording pipettes were pulled from filamented borosilicate glass (Sutter Instruments) to a tip resistance of 3-8 M $\Omega$ using a DMZ Zeitz-Puller (Zeitz). Electrophysiology, image collection and subsequent analysis were performed using Acq4. Signals were amplified using Multiclamp 700B amplifiers (Molecular Devices) and digitized at 50-200 kHz using ITC-1600 digitizers (Heka). Neurons were held in whole-cell patch clamp with an internal solution containing (in mM): $130 \mathrm{~K}$-gluconate, $10 \mathrm{HEPES}, 0.3$ ethylene glycol-bis $(\beta$ aminoethyl ether)-N,N,N',N'-tetraacetic acid (EGTA), 3 KCl, 0.23 Na2GTP, 6.35 Na2Phosphocreatine, 3.4 MgATP, 13.4 biocytin, and $50 \mu \mathrm{M}$ Cascade Blue dye.

Voltron $_{585}$-associated fluorescence was examined using a $595 \mathrm{~nm}$ LED (Thorlabs) at $6.9 \mu \mathrm{W} / \mathrm{mm}^{2}$ power and $598 / 25 \mathrm{~nm}$ excitation and 650/54 nm emission filters (Semrock). Images were collected by sampling a $675 \mu \mathrm{m}$ $\times 137 \mu \mathrm{m}$ region of the slice with a digital sCMOS camera (Hamamatsu; Flash $4.0 \mathrm{~V} 2$ ) at $500 \mathrm{~Hz}$ and $4 \times 4$ pixel binning. Image analysis was performed by custom routines written in Python. For each camera frame, average fluorescence intensity over an elliptical region of interest (ROI) over neuropil adjacent to a cell was subtracted from an identically shaped region containing the cell itself. Synthetic post-synaptic potentials (synPSPs) of $15 \mathrm{mV}$ to $+15 \mathrm{mV}$ in $5 \mathrm{mV}$ increments, repeated for a total of 10 trials per cell were injected in voltage clamp mode. Two adjacent $10 \mathrm{~ms}$-long temporal windows prior to the onset of the current injection were designated as "noise" and "baseline" epochs, and a third $10 \mathrm{~ms}$ "signal" temporal window was centered over the fluorescence peak (from $4 \mathrm{~ms}$ to $14 \mathrm{~ms}$ after the onset of the change in membrane potential). The $\Delta F / F$ was calculated for each trial as the average change in fluorescence between the "signal" and "baseline" windows. To determine SNR, the least-squares regression line for the $\Delta F / F$ was used to determine the average change in signal per $\mathrm{mV}$. The noise for this ratio was calculated by determining the standard deviation of the $\mathrm{dF} / \mathrm{F}$ between the "noise" and "baseline" windows (when the membrane potential in the cell was held constant), and then dividing the signal per $\mathrm{mV}$ by that value. Thus, a value of 0.5 indicates that the fluorescence change associated with a $4 \mathrm{mV}$ alteration in membrane potential is equal in magnitude to $2 \times$ the st.dev. of fluorescence values during a period in which the membrane potential is unchanged.

\section{Simultaneous voltage imaging and optogenetic stimulation in brain slices}

Voltron2 and Channelrhodopsin2 were expressed throughout the motor cortex using injections of a mixture of (1) rAAVretro-hSyn-Cre-WPRE (2x1099 g.c.; Addgene \#105553-AAVrg), (2) AAV1-Syn-FLEX-Voltron2 ${ }_{585}-$ WPRE

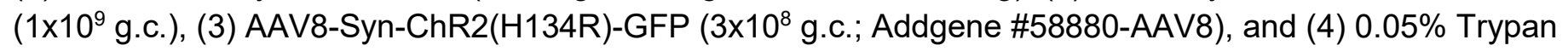
Blue in $1 \mu \mathrm{L}$ of sterile PBS into the lateral ventricle of C57BI/6N mice (Charles River) at postnatal day 1 (Kim et al. 2014). At least 14 days following virus injection, mice were transcardially perfused with $15 \mathrm{~mL}$ of chilled and carbogen-bubbled $\left(95 \% \mathrm{O}_{2} / 5 \% \mathrm{CO}_{2}\right) \mathrm{NMDG}$ aCSF solution (in mM: $92 \mathrm{NMDG}, 2.5 \mathrm{KCl}, 1.25 \mathrm{NaH}_{2} \mathrm{PO}_{4}, 30$ $\mathrm{NaHCO}_{3}, 20 \mathrm{HEPES}, 25$ glucose, 2 thiourea, $5 \mathrm{Na}$-ascorbate, $3 \mathrm{Na}$-pyruvate, $0.5 \mathrm{CaCl}_{2} \cdot 4 \mathrm{H}_{2} \mathrm{O}$ and 10 $\left.\mathrm{MgSO}_{4} \cdot 7 \mathrm{H}_{2} \mathrm{O}, \mathrm{pH} 7.3-7.4,300-310 \mathrm{mOsm}\right)$. Acute slices through motor cortex were made in chilled NMDG 
aCSF with constant bubbling (Ting et al. 2014). Following re-introduction of sodium in $37^{\circ} \mathrm{C}$ NMDG aCSF, slices were transferred to a holding chamber containing $25 \mathrm{nM} \mathrm{JF}_{585}$ dye in $5 \mathrm{~mL}$ bubbled, room temperature HEPES aCSF buffer (in mM: $92 \mathrm{NaCl}, 2.5 \mathrm{KCl}, 1.25 \mathrm{NaH}_{2} \mathrm{PO}_{4}, 30 \mathrm{NaHCO}_{3}, 20 \mathrm{HEPES}, 25$ glucose, 2 thiourea, $5 \mathrm{Na}$-ascorbate, $3 \mathrm{Na}$-pyruvate, $2 \mathrm{CaCl}_{2} \cdot 4 \mathrm{H}_{2} \mathrm{O}$ and $2 \mathrm{MgSO}_{4} \cdot 7 \mathrm{H}_{2} \mathrm{O}$, pH 7.3-7.4, 300-310 mOSm). Slices were incubated in dye solution for 1 hour, and moved to fresh HEPES aCSF for 1 hour to wash out excess dye. Experiments were performed at room temperature in HEPES aCSF solution. Whole-cell recordings were made using filamented glass pipettes (Sutter \#BF150-86-10) pulled to 3-8 MOhm resistance (Sutter P-1000 Micropipette Puller), and intracellular recording buffer containing (in $\mathrm{mM}$ ) $145 \mathrm{~K}$-Gluconate, 10 HEPES, 1 EGTA, 2 Mg-ATP, $0.3 \mathrm{Na}_{2}$-GTP, and $2 \mathrm{MgCl}_{2}$ ( $\mathrm{pH} 7.3$, 290-300 mOsm). A patch-clamp headstage (Molecular Devices \#1-CV-7B) mounted on a motorized 4-axis Siskiyou MX7600 manipulator, and Axon Instruments MultiClamp 700b amplifier were used for all recordings.

Imaging was performed using a custom-built confocal microscope at a frame rate of $458 \mathrm{~Hz}$ using a $16 \mathrm{X} / 0.8$ NA water-immersion objective lens (Nikon CFI75 LWD 16X W). High frame rates were achieved using a system similar to that described previously (Badon et al. 2019) but with a 128-facet polygonal scanner (Cambridge Technology SA34) substituted for the $\mathrm{x}$-axis scanner. Voltron2 585 was excited with a $561 \mathrm{~nm}$ laser diode (Vortran Stradus). The time-averaged irradiance at the sample was $33 \mathrm{~mW} / \mathrm{mm}^{2}$ and fluorescence was epi-collected with a dichroic mirror and emission filter (Chroma T570lpxr and ET570Ip), and detected with a silicon photomultiplier (Hamamatsu S14420-1550MG, $\mathrm{V}_{\mathrm{BIAS}}=50 \mathrm{~V}$ ) and amplified on-board with a custom circuit (https://github.com/tweber225/simple-sipm). A blue LED (Thorlabs M470L4) was used to provide fullfield ChR2 stimulation. The LED was filtered and coupled into the confocal beam path with an excitation filter and dichroic mirror (Thorlabs MF475-35 and DMLP505R). Additionally, the LED was attenuated such that the desired irradiance levels $\left(10-50 \mu \mathrm{W} / \mathrm{mm}^{2}\right)$ were within the analog control range of the LED driver (Thorlabs LEDD1B). Image acquisition and stimulus timing were managed with Scanlmage (Pologruto et al. 2003) and WaveSurfer (https://wavesurfer.janelia.org/).

\section{Lattice lightsheet imaging in zebrafish}

In vivo light sheet microscopy of zebrafish was performed as previously described (Liu et al. 2018). Briefly, zebrafish transgenic lines expressing soma-tagged Voltron (Tg[vglut2a:Gal4; UAS:Voltron $\left.\left.{ }_{552}-\mathrm{ST}\right]\right)$ and Voltron2 (Tg[vglut2a:Gal4;UAS:Voltron2552-ST]) were generated. At three days post-fertilization (dpf), fish were incubated in a water solution containing $3 \mu \mathrm{M} \mathrm{JF}_{552}$ for $2 \mathrm{~h}$. The fish at 4 to $6 \mathrm{dpf}$ were then paralyzed by abungarotoxin $(1 \mathrm{mg} / \mathrm{mL})$ and mounted in low melting point agarose for imaging. The custom microscope used for imaging was described previously (Liu et al. 2018). Here it was used without the adaptive optics (AO) subsystem since optical aberration was negligible in the structure we imaged. A $740 \mathrm{~nm}$ thick light sheet was created from a $560 \mathrm{~nm}$ laser source using a multi-Bessel lattice with an outer and inner NA of 0.38 and 0.36 , respectively, for a measured power of $100 \mu \mathrm{W}$ at the back pupil of the excitation objective. Single-plane imaging was performed at an effective $108 \times 108 \mathrm{~nm}$ XY resolution, with an FOV of 256x512 pixels, at a framerate of $400 \mathrm{~Hz}$. Approximately 4-10 Voltron-expressing neurons were present in each field of view. Fluorescent signal was recorded for $5 \mathrm{~min}$. For analysis, the automated voltage imaging analysis package Volpy was used (Cai et al. 2020). To perform an unbiased comparison of Voltron ${ }_{552}$ and Voltron $2_{552}$ populations, every spiking cell detected by Volpy was included in the analyzed dataset, irrespective of AP 
amplitude. $\triangle F / F_{0}$ and SNR for each cell were calculated by Volpy. For SNR calculations, the noise was defined as the standard deviation of the residual after subtracting spike and subthreshold components, as detected by Volpy.

\section{Voltron imaging in adult flies}

Experiments were performed as described previously (Abdelfattah et al. 2019). Briefly, crosses of Voltron ${ }_{552}$ (UAS-IVS-syn21-Ace2NHalo-p10 Su(Hw)attP8) or Voltron2 552 (UAS-IVS-syn21-Ace2N(A122D)Halo-p10 $\mathrm{Su}(\mathrm{Hw})$ attP8) reporters with split Gal4 drivers were raised on standard cornmeal food supplemented with alltrans-retinal $(0.2 \mathrm{mM}$ before eclosion and then $0.4 \mathrm{mM})$. 2- to 10-day old female progeny were collected for experiments. To prepare the fly for imaging, a small hole was dissected in the head capsule, and air sacs and fat tissue were removed but we did not intentionally remove the perineural sheath. The exposed brain was then bathed in a drop ( $200 \mu \mathrm{L})$ of dye-containing saline $\left(1 \mu \mathrm{M}\right.$ for $\mathrm{JF}_{552}$-Halotag ligand) for $1 \mathrm{hr}$. Saline contains (in $\mathrm{mM}$ ): $\mathrm{NaCl}, 103 ; \mathrm{KCl}, 3 ; \mathrm{CaCl}_{2}, 1.5 ; \mathrm{MgCl}_{2}, 4 ; \mathrm{NaHCO}_{3}, 26$; $\mathrm{N}$-tris(hydroxymethyl)methyl-2aminoethanesulfonic acid, 5; $\mathrm{NaH}_{2} \mathrm{PO}_{4}$, 1; trehalose, 10; glucose, $10\left(\mathrm{pH} 7.3\right.$ when bubbled with $95 \% \mathrm{O}_{2}$ and $\left.5 \% \mathrm{CO}_{2}, 275 \mathrm{mOsm}\right)$. The dye was then washed-out by rinsing three times with $\sim 10 \mathrm{~mL}$ of fresh saline each time over a 1-hr period. Imaging was performed on a widefield fluorescence microscope (SOM, Sutter Instruments) equipped with a 60x, NA 1.0, water-immersion objective (LUMPlanFI/IR; Olympus) and an sCMOS camera (Orca Flash 4.0 V3, Hamamatsu). Images were acquired at 800 frames per second with $4 \times 4$ binning through the Hamamatsu imaging software (HClmage Live). For $\mathrm{JF}_{552}$, illumination was provided by a 561-nm LED (SA-561-1PLUS, Sutter) with an excitation filter (FF01-549/12-25, Semrock); intensity at the sample plane was $2-11 \mathrm{~mW} / \mathrm{mm}^{2}$ for typical recordings. Emission was separated from excitation light using a dichroic mirror (FF562-Di03-25x36, Semrock) and an emission filter (FF01-590/36-25, Semrock). We found that $\mathrm{JF}_{552}$ allows for longer-duration imaging compared with $\mathrm{JF}_{549}$ and $\mathrm{JF}_{525}$, which we used previously (Abdelfattah et al. 2019). At the aforementioned illumination levels, spiking activity was detectable for over 20 min in PPL1-y1pedc and over 10 min in MBON-y1pedc $>\alpha / \beta$.

For MBON- $\gamma 1$ pedc $>\alpha / \beta$, both left and right hemispheres were sampled, while for PPL1- $\gamma 1$ pedc, whose axons project bilaterally, only one hemisphere was imaged. Each experiment at one illumination level consists of a recording of $15 \mathrm{~s}$. Data were analyzed with custom-written scripts in MATLAB (Mathworks). Regions of interest (ROIs) corresponding to the $\mathrm{y} 1$ region were manually selected, and the mean pixel intensity within the ROI was calculated. The raw fluorescence trace was de-trended by median filtering with a $50 \mathrm{~ms}$ time window. $\mathrm{F}_{0}$ was calculated from the filtered trace as the mean over the first $1 \mathrm{~s}$ of imaging session. Spike sorting and SNR quantification were performed on the de-trended trace. Spikes were automatically detected by finding local minima and verified by visual inspection. SNR was quantified as peak amplitude over the standard deviation of the trace excluding a $50 \mathrm{~ms}$ time window around any spikes.

\section{Imaging Parvalbumin (PV) neurons in mouse hippocampus}

Hippocampal PV neuron imaging was performed using adult PV-Cre mice (JAX 008069). The imaging window was implanted using procedures similar to those previously described (Dombeck et al. 2010). In short, a 
circular craniotomy ( $3 \mathrm{~mm}$ diameter) was made centered at $2.0 \mathrm{~mm}$ caudal and $2.0 \mathrm{~mm}$ lateral to bregma. The surface of CA1 was exposed by gently removing the overlying cortex with aspiration. AAV2/1-syn-FLEXVoltron $_{552-S T}$ (Voltron $_{552} ; 4$ mice) and either AAV2/1-syn-FLEX-Voltron2 $552-S T$ (2 mice) or AAV2/1-CAG-FLEXVoltron2 ${ }_{552}$-ST (Voltron2552, 3 mice) virus was diluted to $1.4 \times 10^{13}, 4.1 \times 10^{13}$ and $8.26 \times 10^{11} \mathrm{GC} / \mathrm{mL}$, respectively. Diluted viruses were injected at four locations (separated by $700 \mu \mathrm{m}, 50 \mathrm{~nL}$ per location) at a depth of $200 \mu \mathrm{m}$ from CA1 surface (injection rate, $1 \mathrm{~nL} / \mathrm{s}$ ). The imaging window (constructed by gluing a $3 \mathrm{~mm}$ diameter cover glass to a stainless steel cannula of $3 \mathrm{~mm}$ diameter and $1.5 \mathrm{~mm}$ height) was placed onto the hippocampus and glued to the skull using super-bond C\&B (Sun Medical). A titanium head bar was glued to the skull for head fixation during imaging.

Imaging experiments started 3 weeks after surgery. $100 \mathrm{nM}$ of $\mathrm{JF}_{552}$ were dissolved in $20 \mu \mathrm{L}$ of DMSO (Sigma) and diluted in $20 \mu \mathrm{L}$ Pluronic ${ }^{\mathrm{TM}} \mathrm{F}-127$ (20\% w/v in DMSO, P3000MP, Invitrogen) and in $80 \mu \mathrm{L}$ PBS. The dye solution was delivered using retro-orbital injection (Yardeni et al. 2011) with a 30 gauge needle. Three hours after dye injection, animals were placed under the microscope and labeled PV+ cells (47 - $137 \mu \mathrm{m}$ deep) were illuminated using a $532 \mathrm{~nm}$ laser (Opus 532, Laser Quantum) through an excitation filter (FF02-520-28, Semrock). Fluorescence was collected using a 16X/0.8 NA objective (Nikon), separated from excitation light using a dichroic mirror (540lpxr, Chroma) and an emission filter (FF01-596/83, Semrock), and imaged onto a CMOS camera (DaVinci-1K, RedShirt) using a 50mm camera lens (Nikkor 50mm f1.2, Nikon) as the tube lens. For patterned illumination, the laser beam was expanded using a pair of lenses (C280TMD-A and AC254-150A, Thorlabs) and directed to a digital micromirror device or DMD (V7000, ViALUX). The DMD was imaged to the sample using an $80 \mathrm{~mm}$ lens (AC254-080-A, Thorlabs) and the microscope objective. A reference image of labeled cells was first acquired using widefield illumination. Bright and in-focus neurons were selected manually and their coordinates were used to generate an illumination mask consisting of $64 \mu \mathrm{m}$ diameter discs centered on each selected cell. The illumination intensity was $\sim 70-140 \mathrm{~mW} / \mathrm{mm}^{2}$ (i.e. $\sim 0.22-0.45 \mathrm{~mW}$ per cell) at the sample plane. Images (190 x 160 pixels, corresponding to an area of $1.4 \times 1.2 \mathrm{~mm}$ ) were collected at $2000 \mathrm{~Hz}$ using Turbo-SM64 software (Sci-Measure) for three minutes (360,000 images).

Brain motion was corrected using rigid registration. The fluorescence $F(t)$ of each cell was measured by averaging pixel values within a 10-pixel region covering the cell body. To correct for bleaching and other slow fluctuations, a baseline fluorescence trace $F_{0}(t)$ was computed from $F(t)$ by a moving average with $1 \mathrm{~s}$ windows. Since Voltron fluorescence decreases with membrane depolarization, we define $\Delta F / F_{0}(t)=(-(F(t)-$ $\left.\left.\mathrm{F}_{0}(\mathrm{t})\right)\right) /\left(\mathrm{F}_{0}(\mathrm{t})\right)$ as an estimate of cells' membrane potential. To detect APs, a high pass filtered version of $\Delta \mathrm{F} / \mathrm{F}_{0}$, $\left(\Delta \mathrm{F} / \mathrm{F}_{0}\right)_{\mathrm{hp}}$, was computed by subtracting a median-filtered $\left(5 \mathrm{~ms}\right.$ window) $\Delta \mathrm{F} / \mathrm{F}_{0}$. Positive peaks of the $\left(\Delta \mathrm{F} / \mathrm{F}_{0}\right)_{\mathrm{hp}}$ trace were detected and considered as candidate spike locations (with $t_{k}$ and $p_{k}$ being the locations and the amplitudes, respectively, of the $k^{\text {th }}$ candidate peak). To choose a threshold, the distribution of $p_{k}, P(x)$, was estimated by kernel density method ('ksdensity' function in MATLAB). The same procedure was applied to the inverted $\left(\Delta F / F_{0}\right)_{h p}$ trace to detect 'noise' peaks, and the amplitudes of those peaks were used to construct a noise distribution, $P_{\text {noise }}(x)$. The distribution of spike amplitudes was estimated as $S(x)=P(x)-P_{\text {noise }}(x)$, and a threshold value th1 was chosen at the location where $S(t h 1)=P_{\text {noise }}(t h 1)$ in order to minimize the sum of type I and type II error. This approach works well in cells with good signal to noise ratio (SNR), but in low SNR cells it often leads to substantial false positive detections. We estimated the number of false positive detections ( $n F P)$, at any given threshold value, by counting the number supra-threshold 'noise' peaks in the inverted $\left(\Delta F / F_{0}\right)_{h p}$ trace. If $n F P$ at th1 exceeds 18 over the $180 \mathrm{~s}$ recording period (i.e. false positive rate $>0.1 \mathrm{~Hz}$ ), the threshold was replaced by a higher value, th2, that allowed a maximum of 18 false positive detections. Candidate peaks larger than the threshold were used for an initial estimate of spike times, and segments of the $\left(\Delta \mathrm{F} / \mathrm{F}_{0}\right)_{\mathrm{hp}}$ trace around these peaks were averaged to generate an initial estimate of the AP waveform, AP(t). Since AP waveforms exhibit finite rise and decay times, the occurrence of a spike interferes with the detection of spikes 
within its immediate neighborhood. To correct for this effect, if a candidate peak $p_{i}$ was surrounded by a larger peak $p_{j}$ within $\pm 2 \mathrm{~ms}$, its amplitude was corrected by assuming that a spike occurred at $t_{j}$ and by subtracting the contribution of that spike, i.e. $\mathrm{p}_{\mathrm{i}, \text { corrected }}=\mathrm{p}_{\mathrm{i}}-\mathrm{AP}\left(\mathrm{t}_{\mathrm{i}} \mathrm{t}_{\mathrm{j}}\right)$. This procedure was used to correct the amplitudes of all candidate peaks. Finally, a candidate peak was detected as an AP if its corrected amplitude exceeded the above mentioned threshold.

To quantify the recording quality and the fidelity of spike detection, we first estimated the spike amplitude A by averaging the amplitudes of all detected spikes. The noise of the recording $\sigma$ was estimated as the standard deviation of the $\left(\Delta F / F_{0}\right)_{h p}$ trace excluding regions $2 \mathrm{~ms}$ before and $4 \mathrm{~ms}$ after each detected spike. The signal to noise ratio was measured for each cell as $S N R=A / \sigma$. A cell was included into our analysis if (1) its SNR exceeded 5, (2) the number of detected spikes in the cell exceeded 90 (i.e. spike rate $>0.5 \mathrm{~Hz}$ ), (3) less than $1 \%$ of detected spikes had an inter-spike-interval less than $2.0 \mathrm{~ms}$, and (4) the half-width of the spike waveform was shorter than $0.85 \mathrm{~ms}$. To compare the density of labeled neurons, a z-stack of images was acquired at the end of the recording session and cell bodies in a $1280 \times 1280 \times 200 \mu \mathrm{m}^{3}$ volume were counted manually.

\section{Imaging in mouse visual cortex}

All procedures were approved by the Institutional Animal Care and Use Committee at the Second Faculty of Medicine, Charles University in Prague. The procedures were carried out in accordance with the relevant guidelines and regulations.

Layer 2/3 pyramidal neurons in the visual cortex of mice (C57BI/6NCrl; Charles River Laboratories) were sparsely labeled with the indicators - either Voltron ${ }_{525}-\mathrm{ST}$ or Voltron2 ${ }_{525}$-ST. We prepared four mice per group. In an anesthetized mouse (isoflurane in pure oxygen; $4 \%$ for induction, 1-2\% for maintenance), we first glued a ring-shaped titanium headbar to the skull of the animal using a gel-form cyanoacrylate and then fully closed the skin around the headbar. A craniotomy ( $4.5 \mathrm{~mm}$ in diameter) was drilled over the left parietal cortex, centered on $-2.5 \mathrm{~mm}$ lateral, $+0.5 \mathrm{~mm}$ anterior from lambda (visual cortex). Using beveled, pulled-glass capillaries (tip size $<12 \mu \mathrm{m}$ ), we injected a mixture of two viruses: high-titer AAV carrying the cassette for conditional expression of the voltage indicator (AAV2/1-syn-FLEX-Voltron 525 -ST or AAV2/1-syn-FLEXVoltron2 ${ }_{525}$-ST; titer $10^{12} \mathrm{GC} / \mathrm{mL}$ ) and low-titer AAV carrying the transcription permissive signal (AAV9CamKIla-Cre; titer $10^{8} \mathrm{GC} / \mathrm{mL}$ ). Six to eight $40 \mathrm{~nL}$ injections at a depth of $150 \mu \mathrm{m}$ were performed in each mouse. The craniotomy was coversliped and the cranial window was secured using cyanoacrylate. A standard analgesia protocol (ketoprofen) followed. Approximately seven weeks after surgery, the animal was prepared for imaging. One day prior to imaging, $\mathrm{JF}_{525}$ dye was administered intravenously. To prepare the JF dye for injection, $100 \mathrm{nM}$ of lyophilized $\mathrm{JF}_{525}$ was dissolved in $20 \mu \mathrm{L}$ of DMSO, $20 \mu \mathrm{L}$ Pluronic F-127 $(20 \% \mathrm{w} / \mathrm{v}$ in DMSO), and $60-80 \mu \mathrm{L}$ of PBS. Mice were briefly anesthetized and $100 \mu \mathrm{L}$ of the dye solution was injected into the retro-orbital sinus of the right eye using a 30-gauge needle. We used the same design of wide-field fluorescence microscopy with structured illumination as described in (Abdelfattah et al. 2019). Illumination was delivered using a $525 \mathrm{~nm}$ LED (Mightex, LCS-0525-60-22) and shaped using a digital mirror device (Texas Instruments, LightCrafter). The microscope was equipped with a water immersion objective (20X, NA 1.0, Olympus XLUMPLFLN) and a CMOS camera (Hamamatsu Orca Flash v3). Excitation and emission were separated using a standard filter cube (Chroma 49014; excitation 530/30, dichroic 550, emission 575/40). The illumination was restricted to single neurons using DMD. The illuminated spot was $80 \mu \mathrm{m}$ in diameter and the intensity was kept at $18.5 \mathrm{~mW} / \mathrm{mm} 2$ in the sample plane. Small fields of view ( $40 \mu \mathrm{m} \times 40 \mu \mathrm{m})$ containing 
single neurons were typically captured. Native 2048x2048 resolution of the camera was binned by a factor of 4 . During imaging, we recorded only from neurons that produced at least $\sim 120$ photons per frame and per pixel as this was expected to lead to approximately $1 \%$ standard deviation of the raw signal (quantum efficiency of the camera $82 \%$, neuron covered with $\sim 100$ pixels, noise dominated by shot noise). Three-minute time series at 500 frames per second were captured for most of the recordings; one minute at 1000 frames per second was used only for comparison of AP-related fluorescence changes. Mice were imaged fully awake without any visual stimulation.

To process the recordings, we first removed the in-plane motion artifacts using the fast rigid registration algorithm NoRMCorre (Pnevmatikakis and Giovannucci 2017). Neurons ( $n=107$ expressing Voltron $525-S T$ in 4 mice, $n=102$ expressing Voltron $2_{525}-S T$ in 4 mice) were then segmented manually. The signal was taken as the mean intensity over the region of the interest. The in vitro data showed a substantial difference in brightness of the two indicators. Since voltage-independent background autofluorescence (presumed to also be independent of the chosen indicator) would comprise different fractions of the signal and decrease the observed relative fluorescence changes, we subtracted the mean intensity of the neuropil surrounding each particular neuron from its signal $\left(I_{n}\right)$. To detrend the signal and extract the fluorescence changes related to both APs and slower membrane voltage changes (EPSPs, oscillations), we calculated a baseline ( $\mathrm{B}_{5 \mathrm{~s}}$ ) using a $5 \mathrm{~s}$ median filter. The $\Delta F / F$ trace was then defined as $\Delta F / F=100 *\left(\mathrm{I}_{n}-\mathrm{B}_{5 \mathrm{~s}}\right) / \mathrm{B}_{5 \mathrm{~s}}$. To extract only the AP-related fluorescence spikes, we calculated another baseline $\left(B_{20}\right)$ using a 20 ms median filter; $\Delta F / F_{A P s}=100 *\left(I_{n}\right.$ $\left.\mathrm{B}_{20 \mathrm{~ms}}\right) / \mathrm{B}_{20 \mathrm{~ms}}$. We estimated the noise directly from the $\Delta F / F_{A P s}$ trace. Based on the fact that the AP-related spikes are all negative-going and APs are generally sparse, positive values of the trace $\Delta F / F_{A P s}$ can be considered as noise. We removed all negative data points and then randomly assigned positive/negative signs to the rest of the points. We calculated the standard deviation of these values $\left(S D_{\text {noise }}\right)$ for each neuron and set

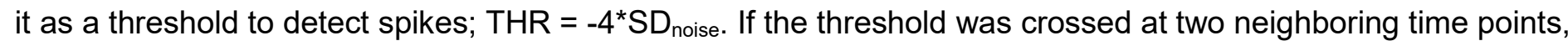
such doublet was considered as a single AP, the time point with higher amplitude was chosen by the algorithm and the spike was ascribed to this time point. Using four standard deviations leads to false positivity rate of 12 false spikes per minute in our recordings.

To detect the periods of $3-5 \mathrm{~Hz}$ oscillations, we applied a bandpass filter ( $3-5 \mathrm{~Hz}, \mathrm{MATLAB}$ [bandpass]) to the $\Delta F / F$ trace and then detected the pronounced oscillations as outliers from the values' variance. The bandpassed trace was thresholded by $3^{*}$ MAD (median absolute deviations) using the MATLAB function [isoutlier]. Absolute values of these outliers were averaged for each neuron and later averaged over all neurons in both groups.

\section{Results}

\section{High throughput screening of Voltron mutants in neuron culture}

Voltron variants were generated using site saturation mutagenesis (SSM) performed at 40 positions within the rhodopsin domain. All screening was performed on Voltron mutants labeled with $\mathrm{JF}_{525}\left(V_{\text {Voltron }}{ }_{25}\right.$ ). Positions were chosen based on: (1) previous reports of analogous positions in other opsins that affected their thermal stability (Curnow et al. 2011; Faham et al. 2004; Mclsaac et al. 2014; Perálvarez-Marín et al. 2008; Wagner et al. 2013), (2) amino acids in close proximity to the retinal chromophore that we reasoned might affect the 
environment of the Schiff base, or (3) positions that were found to be important in mutagenesis of Archaerhodopsin into a voltage sensor (Hochbaum et al. 2014) (Fig 1a). We performed two rounds of screening (Fig. 1b). In the first, we screened individual point mutations using a field stimulation assay in primary neuron cultures (Fig. 1C-e). For each variant, parameters relevant to the performance of the sensor in vivo were measured: AP sensitivity $\left(\triangle \mathrm{F} / \mathrm{F}_{0}\right)$, AP rise and decay kinetics $\left(\tau_{O N}\right.$ and $\left.\tau_{O F F}\right)$, and baseline fluorescence $\left(F_{0}\right)$. To control for biological variability, the measured parameters of each construct were also normalized to an in-plate Voltron ${ }_{525}$ control. The control was also used to monitor the quality and consistency of the screen. For a construct screened in a 96-well plate, results were discarded if at least one of the following quality control $(\mathrm{QC})$ criteria (empirically determined) were violated: (1) the average $\left|\Delta F / F_{0}\right|$ of the in-plate Voltron $_{525}$ controls was $<3.6 \%$, (2) the PDI of the plate was $>30 \%$ (see Methods), or (3) the construct had < 100 pixels with a significant change in $\Delta \mathrm{F} / \mathrm{F}_{0}$ during the stimulation ("responsive pixels").

Of the 2,727 variants that were screened in 199 plates, 2,314 (84\%) passed the above QC criteria. Variants that failed QC were re-screened again and 34\% of them passed QC on the second round of screening and were added to the main QC-passed pool. The majority (66\%) of the libraries were then sequenced and results from the same mutation were grouped, resulting in 819 QC-passing mutants. We found 422 mutants (51\%) with significantly improved $\Delta \mathrm{F} / \mathrm{F}_{0}, 310$ mutants (38\%) with increased SNR, 233 mutants (27\%) with reduced $\tau_{O N}, 256$ mutants $(31 \%)$ with reduced $\tau_{O F F}$, and 307 (37\%) with increased $F_{0}$ compared to Voltron (Fig. 1f, $\mathrm{P}<0.01$, Mann-Whitney $U$ test). The key feature of Voltron we desired to optimize was $\Delta \mathrm{F} / \mathrm{F}_{0}$; therefore, we ranked all variants based on $\left|\Delta F / F_{0}\right| \max$ (maximum of $\left|\Delta F / F_{0}\right|$ ) normalized to in-plate Voltron controls.

Although many variants had improved $\left|\Delta F / F_{0}\right|_{\max }$ over Voltron $_{525}$, there was no single top-performing variant in this first round of screening. Instead, the difference in $\left|\Delta F / F_{0}\right|_{\max }$ of the top 3 variants was only $\sim 10 \%$, which was under our PDI metric (14 $\pm 5.2 \%$ across the first screening round), indicating that the ranking of the top variants may not be accurate. The top two hits in the screen were Voltron ${ }_{525} . \mathrm{V} 74 \mathrm{G}\left(\left|\Delta \mathrm{F} / \mathrm{F}_{0}\right|_{\max }\right.$ relative to Voltron $\left._{525}=2.28\right)$ and Voltron ${ }_{525} . V 74 \mathrm{~W}\left(\left|\Delta \mathrm{F} / \mathrm{F}_{0}\right|_{\max }\right.$ relative to Voltron ${ }_{525}=2.21$; Fig. $1 \mathrm{~g}$, Supplementary Table 1). However, subsequent analysis with patch-clamp revealed that Voltron ${ }_{525} . A 122 D$ ( $3^{\text {rd }}$ in the ranked $\left|\Delta F / F_{0}\right|_{\text {max }}$ list, $\left|\Delta \mathrm{F} / \mathrm{F}_{0}\right|_{\max }$ relative to Voltron $\left.{ }_{525}=2.18\right)$ had superior properties as a voltage sensor. The Voltron ${ }_{525} . \mathrm{A} 122 \mathrm{D}$ mutant (which we named Voltron2 ${ }_{525}$ ) exhibited $\left|\Delta \mathrm{F} / \mathrm{F}_{0}\right|_{\max }$ and $\mathrm{SNR}$ that was $52 \%$ and $25 \%$, respectively, higher than Voltron 525 (Fig. 1 h,i).

The first-round SSM screen revealed many mutations that moderately increased $\Delta F / F_{0}$. We therefore embarked on a second round of combinatorial (combo) screening, hoping that combining 13 of the top performing mutations (Y63L, N69E, V74E/W, R78H, N81S, L89A/C/G/T, A122D/H, V196P) would further improve the sensor. Of the 1,232 constructs screened in 106 plates, $77 \%$ passed QC. Surprisingly, only 28 of 848 combo mutants $(3.3 \%)$ had significantly improved $\left|\Delta F / F_{0}\right|_{\max }$ over Voltron2 ${ }_{525}(P<0.01$, Mann-Whitney $U$ test; Supplementary Fig. 1, Supplementary Table 2). Similarly, only a few variants had increased SNR (20 of $848,2.4 \%$ ). The A122D substitution was present in $34 \%$ of the combo variants passing QC (Supplementary Table 3); nevertheless, the combo screen revealed that combining it with other mutations resulted in less sensitive variants. Subsequent automated patch-clamp analysis confirmed that Voltron ${ }_{525}$, containing the sole A122D substitution, outperformed all combo mutants (Supplementary Fig. 4). 


\section{Screening and characterization with automated whole-cell electrophysiology}

Many single and combo mutation hits from the neuron culture screen had improved $\left|\Delta F / F_{0}\right|_{\max }$ over Voltron but had very similar $\Delta F / F_{0}$ characteristics among them. We deemed the field stimulation screen to be insufficiently sensitive to find the one variant with the best performance, so we used the uM Workstation, a fully automated whole-cell electrophysiology platform based on the PatcherBot to perform a secondary screen on top single and combinatorial mutant hits.

We first validated the throughput and performance of the automated electrophysiology platform. To mimic a small-scale screen, $1035-\mathrm{mm}$ Mattek dishes of cultured neurons were transfected with variants of the voltage sensor ASAP (St-Pierre et al. 2014). The uM Workstation made 103 patch-clamp attempts in 7.1 hours, with a $78 \%$ whole-cell success rate. The system operated unattended for $\sim 5$ hours during that day of screening. Thus, the uM Workstation allowed us to screen $\sim 10$ constructs per day, assuming 5-10 neurons per construct.

The uM Workstation achieves high throughput by automatically cleaning and reusing patch-clamp pipettes (Fig. 2a); however, it is conceivable that the cleaning process is imperfect and whole-cell success rate degrades over subsequent attempts. To address this, we evaluated pipette performance after multiple patchclamp attempts. Whole-cell success rate decreased over time, but likely due to cell health degradation, not due to an accumulation of debris on the reused pipette, since replacing the pipette did not recover the success rate (Supplementary Fig. 2a). In a separate experiment we replaced the dish without replacing the pipette, and found that the success rate recovered, further suggesting that cell health degradation, not pipette debris is responsible for the apparent decrease in success rate (Supplementary Fig. 2b). To explore the limits of pipette cleaning, we patch-clamped cells with the same pipette, replacing the plate as needed, until the time to form a gigaohm seal increased, indicating a contaminated pipette. Consistent with previous observations, a single pipette could be used for patch-clamping $~ 50$ neurons (Kolb et al. 2019) (Supplementary Fig. 2c). Last, we evaluated the quality of the recordings and found $85.6 \%$ (143 out of 167) of the successful whole-cell recordings had a holding current greater than $-100 \mathrm{pA}$ and access resistance less than $30 \mathrm{M} \Omega$, which meets the criteria for most of the published data acquired with manual patch clamp. Together, we found that the automated uM Workstation successfully increased our throughput, enabling large-scale patch-clamp studies, without compromising data quality.

Using the uM Workstation we then screened top-performing single-position mutants from the field stimulation screen, including Voltron as a control. While Voltron ${ }_{525}$.V74G and Voltron 525. V74W were the top performers from the field stimulation screen, their fluorescence response to a $100 \mathrm{mV}$ voltage step was lower than that of Voltron2525 (Fig. 2b). The other mutants were also $8 \%$ to $55 \%$ less sensitive to $100 \mathrm{mV}$ voltage steps than Voltron2 (Supplementary Fig. 3). Meanwhile, Voltron2 ${ }_{525}$ was found to be $65 \%$ more sensitive than Voltron, consistent with the field stimulation screen. Furthermore, in the physiologically relevant sub-threshold voltage range $(-90$ to $-50 \mathrm{mV})$, Voltron2 525 exhibited a significantly steeper slope than Voltron ${ }_{525}(0.54 \pm 0.01$ and $0.21 \pm 0.01 \% / \mathrm{mV}$, respectively; $\mathrm{P}=0.0009$, Mann-Whitney $U$ test), making it a higher-fidelity optical reporter of changes in sub-threshold membrane potential.

Surprisingly, the combo mutation screen (second round of the field stimulation assay, Fig. 1b) yielded few variants with improved sensitivities. We nevertheless screened the 34 variants with sensitivities marginally better than Voltron2 ${ }_{525}$ using the uM Workstation. As was the case with the single-position mutants, we found no combo mutants that out-performed Voltron2 ${ }_{525}$ (Fig. 2c, Supplementary Fig. 4). Therefore, for the remainder of this study, we focused on characterization of Voltron $2_{525}$. 
Voltron2 ${ }_{525}$ exhibited fast onset and decay kinetics that were best fit with a double exponential (Fig. 2d). Interestingly, the A122D mutation completely eliminated the transient peak in the fluorescence response of Voltron $_{525}$ (Fig. 2b inset). The fast component of the onset and decay kinetics was slightly shorter for Voltron2 525 (onset: $0.67 \pm 0.03 \mathrm{~ms}$, decay: $0.89 \pm 0.09 \mathrm{~ms}$ ) compared to Voltron ${ }_{525}$ (onset: $0.85 \pm 0.06 \mathrm{~ms}$, decay: $1.13 \pm 0.08 \mathrm{~ms}$ ), though not significantly different. The slow components were likewise similar between the two sensors (Voltron 525 : onset 3.26 $\pm 0.47 \mathrm{~ms}$, decay $6.27 \pm 1.41 \mathrm{~ms}$; Voltron2 525 : onset $4.76 \pm 0.92 \mathrm{~ms}$, decay $4.74 \pm 0.32 \mathrm{~ms})$. The fast component of Voltron2 525 accounted for a larger percentage of the overall response in the onset but not decay response (Fig. 2e). Overall, the kinetic properties of Voltron ${ }_{525}$ and Voltron $2_{525}$ were found to be similar.

Consistent with the improved sensitivity of Voltron2 ${ }_{525}$ in response to voltage steps, it was also superior in its sensitivity to APs. Voltron2 525 reported single APs with $\Delta F / F_{0}$ of $10.09 \pm 1.47 \%$, significantly higher than for Voltron $_{525}(6.16 \pm 0.74 \%$, Fig. $2 \mathrm{f}, \mathrm{g})$. The baseline fluorescence of Voltron $2_{525}$ was $\sim 30 \%$ lower than Voltron T25 $_{5}$, which may be beneficial in some experiments but detrimental in others (Fig. $2 \mathrm{~h}$ ). The same trend was observed with the addition of the soma localization tag (Supplementary Fig. 5). Nevertheless, both Voltron $2_{525}$ and Voltron2 ${ }_{525}$-ST showed good membrane localization, qualitatively similar to their Voltron counterparts (Supplementary Fig. 6,7 ). The addition of a soma localization tag to Voltron2 525 increased its sensitivity to a $100 \mathrm{mV}$ depolarization pulse by $\sim 18 \%$ (Supplementary Fig. 8). In culture, Voltron2 525 photobleached slightly, but not significantly, slower than Voltron ${ }_{525}$ (Voltron $_{525}: 45 \pm 2 \%$, Voltron $2_{525}: 41 \pm 1 \%$ reduction in fluorescence; $\mathrm{P}=0.11$, Mann-Whitney $U$ test; Fig. 2i).

We reasoned that the $A 122 D$ mutation responsible for the increased sensitivity of Voltron2 ${ }_{525}$ could have beneficial properties when grafted onto other Ace rhodopsin-based GEVIs. We tested this hypothesis in Ace2N-mNeon and VARNAM. As expected, adding the A122D mutation to both GEVIs increased their sensitivity to depolarizing and hyperpolarizing voltage pulses (Supplementary Fig. 9). Similar to Voltron2 525 , A122D significantly increased the slope of the sensors in the sub-threshold range (Ace2N-mNeon: $0.091 \pm 0.012 \% / \mathrm{mV}$, Ace2N-mNeon.A122D: 0.303 $\pm 0.012 \% / \mathrm{mV}, \mathrm{P}=0.006$; VARNAM: 0.104 $\pm 0.012 \% / \mathrm{mV}$, VARNAM.A122D: 0.147 $\pm 0.010 / \mathrm{mV}, \mathrm{P}=0.045$; Mann-Whitney $U$ test). The mutation eliminated the transient peak from VARNAM but not from Ace2N-mNeon. Grafting A122D onto Positron did not result in increased sensitivity (Supplementary Fig. 10); however this was not surprising given that the proton transport pathway in Positron is different from Voltron (Abdelfattah et al. 2020). Together, the results suggest that the A122D mutation appears to generalize across different FRET donors.

\section{Voltage imaging and stimulation in acute brain slices}

The high sensitivity of Voltron2 in the sub-threshold range of voltages should make it a suitable GEVI for detecting low-amplitude voltage fluctuations, such as those arising as a result of synaptic activity. To test this, synthetic PSPs (synPSPs) were injected into neurons expressing Voltron ${ }_{585}$ and Voltron $2_{585}$ in acute mouse brain slices (Fig. 3a). Optically captured responses to PSPs were $\sim 40 \%$ larger for Voltron $2_{585}$ than Voltron N $_{585}$, consistent with the improved sensitivity of Voltron2 585 in the sub-threshold range (Fig. $3 \mathrm{~b}, \mathrm{C}$ ). The overall $\Delta \mathrm{F} / \mathrm{F}_{0}$ in response to $\pm 15 \mathrm{mV}$ synPSPs was $5.9 \%$ for Voltron $2_{585}$, compared to $3.2 \%$ for Voltron 585 (Fig. $3 \mathrm{~d}$ top). Due to the increased sensitivity, the detectability of synPSPs was found to be significantly improved for Voltron $2_{585}$ (Fig. $3 \mathrm{~d}$ bottom). Together, we found that Voltron2 ${ }_{585}$ could be used to image millivolt-scale synaptic events. 
We then evaluated the ability of Voltron2 to be used in the context of all-optical electrophysiology. Here, we expressed Voltron2 585 -ST along with ChR2-GFP (Boyden et al. 2005) in acute slices of mouse motor cortex (Fig. 4a). We confirmed with whole-cell electrophysiology that ChR2 could reliably elicit spiking activity when illuminated with moderate blue light intensity $\left(30 \mu \mathrm{W} / \mathrm{mm}^{2}\right)$ and that Voltron2 585 accurately tracked the membrane voltage. Increasing the green excitation light intensity improved the SNR as expected but resulted in well-documented cross-excitation issues (Klapoetke et al. 2014; Packer et al. 2015) (Fig. 4b). Using the same illumination intensity, we imaged a FOV with 10 neurons during repeated ChR2 activation and found robust Voltron2 585 signals that reported expected increases in spiking activity during ChR2 stimulation (Fig. $4 \mathrm{c}, \mathrm{d})$. These experiments suggest that Voltron2 ${ }_{585}$ can be used with optogenetic actuators for all-optical interrogation of brain circuitry.

\section{$\underline{\text { In vivo voltage imaging of olfactory sensory neurons in zebrafish }}$}

We next tested Voltron2 ${ }_{552}$ side-by-side with Voltron in olfactory sensory neurons in larval zebrafish using a lattice lightsheet microscope (Fig. 5a). Volton2 ${ }_{552}$ exhibited higher-amplitude spontaneous spiking and subthreshold activity than Voltron ${ }_{552}$ (Fig. $5 b$ ). The $\Delta F / F_{0}$ and SNR of detected spikes was significantly higher for Voltron2 ${ }_{552}$, measured across hundreds of cells (Fig. 5c). Both Voltron2 ${ }_{552}$ and Voltron 552 were imaged over 5 minutes, with voltage signals still clearly visible at the end of the experiment, suggesting that longer recording sessions are also possible.

\section{$\underline{\text { In vivo voltage imaging in adult Drosophila melanogaster }}$}

We tested Voltron2 in voltage recordings of spontaneous activity from two neuron types in the mushroom body $(\mathrm{MB})$ circuit of adult Drosophila melanogaster, the output neuron MBON- $\gamma 1$ pedc $>\alpha / \beta$ and the dopaminergic neuron PPL1-y1pedc (Aso et al. 2014). The expression of Voltron2 was driven by split Gal4 lines (MB112C and $M B 320 C$ ), which uniquely target these neurons, enabling a well-matched comparison of sensor performance across different flies. We imaged both cell types in the $\gamma 1$ compartment, which contains the dendritic processes of MBON- $\gamma 1$ pedc $>\alpha / \beta$ and the axonal terminals of PPL1- $\gamma 1$ pedc. We used JF ${ }_{552}-\mathrm{HaloTag}$ conjugate (Fig. 6a). Among several JF dyes we tried in Drosophila neurons, we found that $\mathrm{JF}_{552}$ allowed for prolonged Voltron imaging, which in PPL1-y1pedc can last over 20 min without significant deterioration of the health of the cell (unpublished observations, $\mathrm{YS}$ ). $\mathrm{JF}_{552}$ is a $\mathrm{JF}_{549}$ analogue with fluorine substitution on the xanthene ring, which shows improved cell and tissue permeability (Zheng et al. 2019). Spike amplitudes $\left(\Delta F / F_{0}\right)$ measured with Voltron2 ${ }_{552}$ were significantly larger when compared to Voltron ${ }_{552}$ (Fig. $\left.6 \mathrm{~b}, \mathrm{~d}\right)$. The mean spike size is $74 \%$ larger in MBON- 1 pedc $>\alpha / \beta$ (Fig. 6c), and $57 \%$ larger in PPL1- $\gamma 1$ pedc (Fig. 6e). The SNR is also increased, most notably in MBON-ү1pedc> $\alpha / \beta$ (Fig. 6f,h). The basal florescence levels are lower with Voltron2 though (Fig. 6g,i), which contributes to the more moderate improvement of SNR as compared to $\Delta \mathrm{F} / \mathrm{F}$. 


\section{In vivo voltage imaging in mouse hippocampus and visual cortex}

We next tested Voltron2-ST in vivo in parvalbumin (PV) expressing interneurons in the CA1 region of the mouse hippocampus. Cells expressing soma-targeted Voltron2 ${ }_{552}-\mathrm{ST}$ and labeled with $\mathrm{JF}_{552}$ were individually illuminated using a DMD-based patterned illumination microscope, and the fluorescence responses of up to 34 neurons were imaged simultaneously at 2000 frames per second (Fig. 7a,b). Spontaneous APs in PV-positive interneurons induced nearly two-fold larger fluorescence changes in Voltron2 552 compared to Voltron 552 expressing neurons (Fig. 7c,d). The baseline fluorescence was dimmer for Voltron2 ${ }_{552}$ (Fig. 7e), leading to slightly larger recording noise (Fig. 7f). Yet the overall SNR was still significantly improved compared to Voltron $_{552}$ (Fig. $7 \mathrm{~g}$ ). The number of visually identifiable neurons was comparable despite the dimmer baseline fluorescence (Supplementary Fig. 11a). Furthermore, photobleaching was significantly slower in Voltron $2_{552}$ compared to Voltron ${ }_{552}$-expressing cells (Supplementary Fig. $11 \mathrm{~b} ; 9.9 \% \pm 5.8 \%$ vs. $15.6 \pm 4.6 \%$ in 3 minutes, respectively).

Voltron2 ${ }_{525}$-ST was then evaluated and benchmarked against Voltron ${ }_{525}-\mathrm{ST}$ in the mouse primary visual cortex. We used one-photon epifluorescence microscopy with structured illumination and the same protocol as previously (Abdelfattah et al. 2019). The chronic cranial window and a mixture of Cre-dependent Voltron and dilute CaMKIla-Cre viruses enabled sparse, but very bright, labeling of pyramidal neurons (Fig. 7h). APs and subthreshold fluctuations were clearly observable using both sensors (Fig. 7i,j). Voltron2 ${ }_{552}$-ST produced larger $\Delta F / F$ responses from spikes in cortical pyramidal neurons (Fig. 7k), and higher imaging rate led to larger responses from both indicators. Similar to other preparations, we observed that Voltron ${ }_{552}$-ST was significantly brighter than Voltron2 ${ }_{552}$-ST (Fig. 7l). As shot noise is dominant in high-speed imaging, we observed smaller relative noise in the brighter Voltron ${ }_{552}$-ST-expressing neurons compared to Voltron2 ${ }_{552}$-ST-expressing neurons (Fig. $7 \mathrm{~m}$ ). There was no significant difference in SNR between the two sensors in this preparation (Fig. 7n), likely because the improved $\Delta F / F$ of Voltron2 552 was offset by its higher noise. We subsequently focused on the improved sensitivity of Voltron 2552 around resting membrane potential. Low-frequency membrane voltage oscillations in individual cortical neurons in awake mice have previously been observed in the barrel (Crochet and Petersen 2006), auditory (Zhou et al. 2014) and visual cortices (Bennett et al. 2013). We focused on brief (1-2 s long) periods of 3-5 Hz oscillations around a $\sim 12 \mathrm{mV}$ hyperpolarized baseline, exhibiting a peak-topeak amplitude of $\sim 17 \mathrm{mV}$ (Einstein et al. 2017). Due to the enhanced sensitivity of Voltron2 ${ }_{552}$ in the subthreshold range, $3-5 \mathrm{~Hz}$ oscillations were significantly more pronounced when imaging Voltron2 ${ }_{552}-\mathrm{ST}$, exhibiting $\sim 50 \%$ larger amplitude (Fig. $7 \mathrm{j}, 0$ ). Together, these data indicate that Voltron2 ${ }_{552}$ significantly improves the quality of in vivo voltage imaging in multiple regions of the mouse brain.

\section{Discussion}

Here we present Voltron2 which contains a mutation to Voltron that increased the sensitivity of the GEVI by > $50 \%$ to APs in culture and in vivo. Moreover, Voltron2 is approximately 3-fold more sensitive to subthreshold changes due to its steeper slope around the resting membrane potential. As with our efforts to engineer positive-going FRET sensors (Abdelfattah et al. 2020), the mutation we discovered generalized to other Acebased GEVIs with fluorescent protein reporters. In both Ace2N-mNeon and VARNAM, the A122D substitution increased sensitivity, particularly in the sub-threshold voltage range. Perhaps the sensitivity-improving mutations identified in our screen will also be useful for optimization of rhodopsin-only GEVIs, such as those based on Arch, that rely on imaging the dim retinal fluorescence directly. 

available under aCC-BY-NC-ND 4.0 International license.

Engineering improved GEVIs has been more challenging than GECls. In this study, we screened $>2,700$ variants to attain $\sim 50 \%$ increase in $\triangle F / F_{0}$ in the Voltron scaffold. Applying the same mutagenesis and screening strategy to GCaMP, RCaMP and R-GECO1 calcium indicators yielded $>500 \%$ increases in $\Delta F / F_{0}$ with $<1,000$ screened variants (Dana et al. 2016, 2019). Further, combining mutations in GCaMP scaffolds has often yielded additive benefits, while doing so in the current context of the Ace2N rhodopsin ultimately did not produce any variants with significant improvements over the best single A122D mutation. It is possible that there are biophysical phenomena that impose a ceiling on the sensitivity of this scaffold. For example, it is expected that the FRET efficiency between the HaloTag-dye or FP donor and the rhodopsin retinal acceptor will limit the maximum fluorescence change. Each of these chromophores resides on or in a bulky protein domain, limiting their closest approach distance. We were intrigued to observe that the A122D mutation improved the sensitivity of the negative-going Voltron, but also decreased its fluorescence at resting membrane potential. It seems possible that additional mutations could restore the original resting fluorescence of Voltron while maintaining the improved sensitivity of A122D, leading to improved SNR, but our screens failed to identify such a variant. Mutations at the equivalent position of Ace2 A122D in bacteriorhodopsin changed the thermal stability of that protein (Wagner et al. 2013).

Various high-throughput platforms have been developed that have been used to screen for improved GEVIs (Chien et al. 2015; Kannan et al. 2018; Park et al. 2013; Piatkevich et al. 2018). The majority of these platforms utilize bacteria or tissue culture cells for screening. We instead opted to perform our high-content primary screen in dissociated neurons, a costlier and more time-consuming strategy, but one that maximized the compatibility of the resulting sensor with in vivo neuronal imaging. Even still, our field stimulation screen was insufficiently sensitive to disambiguate the top-performing sensors. We therefore relied on the automated patch-clamp system that afforded us the ability to screen dozens of sensors faster than possible manually, without compromising data quality. The system had a lower throughput than the field stimulation screen but enabled us to characterize the sensitivity and kinetics of many variants with much higher fidelity. The combination of both field stimulation and patch-clamp screens provided a high-quality assessment of topperforming variants.

We show that like its predecessor, Voltron2 can be readily used for in vivo imaging in mice, flies, and fish, as well as acute brain slice imaging in mice. These experiments generally confirm the characteristics of Voltron2 that we discovered in our cell culture screen; namely increased $\Delta F / F_{0}$ (particularly in the sub-threshold range), improved SNR, reduced baseline fluorescence, and reduced photobleaching in some preparations. These improvements were consistent among the dye ligands that were tested in vivo $\left(\mathrm{JF}_{525}, \mathrm{JF}_{552}\right.$, and $\left.\mathrm{JF}_{585}\right)$. The increased sensitivity of Voltron2 in the subthreshold range was shown to significantly improve the detectability of $10-20 \mathrm{mV}$ oscillations. Given the richness of information contained within subthreshold activity, including excitatory and inhibitory PSPs, oscillations of various frequencies, spikelets, and other features, Voltron2 can be a valuable tool for unveiling neuronal computations in intact preparations. In addition, in multiple preparations, Voltron2 extended the functional imaging time, which will push the limits of the biology that can be studied.

Increasing the sensitivity of GEVIs (the difference in photon flux per millivolt change in membrane potential) and reducing photobleaching still remain the main challenges to increase the adoption of GEVIs for in vivo experimentation. Protein engineering efforts devoted to creating two-photon-compatible GEVIs will also be required to address the emerging trend in the field to image deep in the brain while maintaining single-cell resolution. Chemigenetic indicators like Voltron2 continue to be promising scaffolds to address these goals. 


\section{References}

Abdelfattah AS, Kawashima T, Singh A, Novak O, Liu H, Shuai Y, Huang Y-C, Campagnola L, Seeman SC, Yu J, Zheng J, Grimm JB, Patel R, Friedrich J, Mensh BD, Paninski L, Macklin JJ, Murphy GJ, Podgorski K, Lin BJ, Chen T-W, Turner GC, Liu Z, Koyama M, Svoboda K, Ahrens MB, Lavis LD, Schreiter ER. Bright and photostable chemigenetic indicators for extended in vivo voltage imaging. Science 365: 699-704, 2019.

Abdelfattah AS, Valenti R, Zheng J, Wong A, Podgorski K, Koyama M, Kim DS, Schreiter ER. A general approach to engineer positive-going eFRET voltage indicators. Nat Commun 11: 3444, 2020.

Aso Y, Hattori D, Yu Y, Johnston RM, Iyer NA, Ngo T-T, Dionne H, Abbott L, Axel R, Tanimoto H, Rubin GM. The neuronal architecture of the mushroom body provides a logic for associative learning. eLife 3: e04577, 2014.

Badon A, Bensussen S, Gritton HJ, Awal MR, Gabel CV, Han X, Mertz J. Video-rate large-scale imaging with Multi-Z confocal microscopy. Optica 6: 389-395, 2019.

Bennett C, Arroyo S, Hestrin S. Subthreshold mechanisms underlying state-dependent modulation of visual responses. Neuron 80: 350-357, 2013.

Berg S, Kutra D, Kroeger T, Straehle CN, Kausler BX, Haubold C, Schiegg M, Ales J, Beier T, Rudy M, Eren K, Cervantes JI, Xu B, Beuttenmueller F, Wolny A, Zhang C, Koethe U, Hamprecht FA, Kreshuk A. ilastik: interactive machine learning for (bio)image analysis. Nat Methods 16: 1226-1232, 2019.

Boyden ES, Zhang F, Bamberg E, Nagel G, Deisseroth K. Millisecond-timescale, genetically targeted optical control of neural activity. Nat Neurosci 8: 1263-1268, 2005.

Cai C, Friedrich J, Pnevmatikakis EA, Podgorski K, Giovannucci A. VolPy: automated and scalable analysis pipelines for voltage imaging datasets. bioRxiv 2020.01.02.892323, 2020.

Campagnola L, Kratz MB, Manis PB. ACQ4: an open-source software platform for data acquisition and analysis in neurophysiology research. Front Neuroinformatics 8: 3, 2014.

Chien M-P, A. Werley C, L. Farhi S, E. Cohen A. Photostick: a method for selective isolation of target cells from culture. Chem Sci 6: 1701-1705, 2015.

Chien M-P, Brinks D, Testa-Silva G, Tian H, Brooks FP, Adam Y, Bloxham W, Gmeiner B, Kheifets S, Cohen AE. Photoactivated voltage imaging in tissue with an archaerhodopsin-derived reporter. Sci Adv 7: eabe3216, 2021.

Crochet S, Petersen $\mathrm{CCH}$. Correlating whisker behavior with membrane potential in barrel cortex of awake mice. Nat Neurosci 9: 608-610, 2006.

Curnow P, Di Bartolo ND, Moreton KM, Ajoje OO, Saggese NP, Booth PJ. Stable folding core in the folding transition state of an alpha-helical integral membrane protein. Proc Natl Acad Sci U S A 108: 14133-14138, 2011.

Dana H, Mohar B, Sun Y, Narayan S, Gordus A, Hasseman JP, Tsegaye G, Holt GT, Hu A, Walpita D, Patel R, Macklin JJ, Bargmann Cl, Ahrens MB, Schreiter ER, Jayaraman V, Looger LL, Svoboda K, Kim DS. Sensitive red protein calcium indicators for imaging neural activity. eLife 5: e12727, 2016. 
bioRxiv preprint doi: https://doi.org/10.1101/2021.11.09.467909; this version posted November 11,2021 . The copyright holder for this preprint (which was not certified by peer review) is the author/funder, who has granted bioRxiv a license to display the preprint in perpetuity. It is made available under aCC-BY-NC-ND 4.0 International license.

Dana H, Sun Y, Mohar B, Hulse BK, Kerlin AM, Hasseman JP, Tsegaye G, Tsang A, Wong A, Patel R, Macklin JJ, Chen Y, Konnerth A, Jayaraman V, Looger LL, Schreiter ER, Svoboda K, Kim DS. High-performance calcium sensors for imaging activity in neuronal populations and microcompartments. Nat Methods 16: 649657, 2019.

Dombeck DA, Harvey CD, Tian L, Looger LL, Tank DW. Functional imaging of hippocampal place cells at cellular resolution during virtual navigation. Nat Neurosci 13: 1433-1440, 2010.

Einstein MC, Polack P-O, Tran DT, Golshani P. Visually Evoked 3-5 Hz Membrane Potential Oscillations Reduce the Responsiveness of Visual Cortex Neurons in Awake Behaving Mice. J Neurosci Off J Soc Neurosci 37: 5084-5098, 2017.

Faham S, Yang D, Bare E, Yohannan S, Whitelegge JP, Bowie JU. Side-chain contributions to membrane protein structure and stability. J Mol Biol 335: 297-305, 2004.

Flytzanis NC, Bedbrook CN, Chiu H, Engqvist MKM, Xiao C, Chan KY, Sternberg PW, Arnold FH, Gradinaru V. Archaerhodopsin variants with enhanced voltage-sensitive fluorescence in mammalian and Caenorhabditis elegans neurons. Nat Commun 5: 4894, 2014.

Gibson DG, Young L, Chuang R-Y, Venter JC, lii CAH, Smith HO. Enzymatic assembly of DNA molecules up to several hundred kilobases. Nat Methods 6: 343-345, 2009.

Gong Y, Huang C, Li JZ, Grewe BF, Zhang Y, Eismann S, Schnitzer MJ. High-speed recording of neural spikes in awake mice and flies with a fluorescent voltage sensor. Science aab0810, 2015.

Gong Y, Li JZ, Schnitzer MJ. Enhanced Archaerhodopsin Fluorescent Protein Voltage Indicators. PLOS ONE 8: e66959, 2013.

Grimm JB, English BP, Chen J, Slaughter JP, Zhang Z, Revyakin A, Patel R, Macklin JJ, Normanno D, Singer $\mathrm{RH}$, Lionnet T, Lavis LD. A general method to improve fluorophores for live-cell and single-molecule microscopy. Nat Methods 12: 244-250, 2015.

Grimm JB, Muthusamy AK, Liang Y, Brown TA, Lemon WC, Patel R, Lu R, Macklin JJ, Keller PJ, Ji N, Lavis LD. A general method to fine-tune fluorophores for live-cell and in vivo imaging. Nat Methods 14: nmeth.4403, 2017.

Hochbaum DR, Zhao Y, Farhi SL, Klapoetke N, Werley CA, Kapoor V, Zou P, Kralj JM, Maclaurin D, Smedemark-Margulies N, Saulnier JL, Boulting GL, Straub C, Cho YK, Melkonian M, Wong GK-S, Harrison DJ, Murthy VN, Sabatini BL, Boyden ES, Campbell RE, Cohen AE. All-optical electrophysiology in mammalian neurons using engineered microbial rhodopsins. Nat Methods 11: 825-833, 2014.

Kannan M, Vasan G, Huang C, Haziza S, Li JZ, Inan H, Schnitzer MJ, Pieribone VA. Fast, in vivo voltage imaging using a red fluorescent indicator. Nat Methods 1, 2018.

Kim J-Y, Grunke SD, Levites Y, Golde TE, Jankowsky JL. Intracerebroventricular viral injection of the neonatal mouse brain for persistent and widespread neuronal transduction. J Vis Exp JoVE 51863, 2014.

Klapoetke NC, Murata Y, Kim SS, Pulver SR, Birdsey-Benson A, Cho YK, Morimoto TK, Chuong AS, Carpenter EJ, Tian Z, Wang J, Xie Y, Yan Z, Zhang Y, Chow BY, Surek B, Melkonian M, Jayaraman V, Constantine-Paton M, Wong GK-S, Boyden ES. Independent optical excitation of distinct neural populations. Nat Methods 11: 338-346, 2014.

Kolb I, Landry CR, Yip MC, Lewallen CF, Stoy WA, Lee J, Felouzis A, Yang B, Boyden ES, Rozell CJ, Forest CR. PatcherBot: a single-cell electrophysiology robot for adherent cells and brain slices. J Neural Eng 16: 046003, 2019. 

available under aCC-BY-NC-ND 4.0 International license.

Kolb I, Stoy WA, Rousseau EB, Moody OA, Jenkins A, Forest CR. Cleaning patch-clamp pipettes for immediate reuse. Sci Rep 6: 35001, 2016.

Kralj JM, Douglass AD, Hochbaum DR, Maclaurin D, Cohen AE. Optical recording of action potentials in mammalian neurons using a microbial rhodopsin. Nat Methods 9: 90-95, 2012.

Lin MZ, Schnitzer MJ. Genetically encoded indicators of neuronal activity. Nat Neurosci 19: 1142-1153, 2016.

Liu T-L, Upadhyayula S, Milkie DE, Singh V, Wang K, Swinburne IA, Mosaliganti KR, Collins ZM, Hiscock TW, Shea J, Kohrman AQ, Medwig TN, Dambournet D, Forster R, Cunniff B, Ruan Y, Yashiro H, Scholpp S, Meyerowitz EM, Hockemeyer D, Drubin DG, Martin BL, Matus DQ, Koyama M, Megason SG, Kirchhausen T, Betzig E. Observing the cell in its native state: Imaging subcellular dynamics in multicellular organisms. Science 360, 2018.

Los GV, Encell LP, McDougall MG, Hartzell DD, Karassina N, Zimprich C, Wood MG, Learish R, Ohana RF, Urh M, Simpson D, Mendez J, Zimmerman K, Otto P, Vidugiris G, Zhu J, Darzins A, Klaubert DH, Bulleit RF, Wood KV. HaloTag: A Novel Protein Labeling Technology for Cell Imaging and Protein Analysis. ACS Chem Biol 3: 373-382, 2008.

Mclsaac RS, Engqvist MKM, Wannier T, Rosenthal AZ, Herwig L, Flytzanis NC, Imasheva ES, Lanyi JK, Balashov SP, Gradinaru V, Arnold FH. Directed evolution of a far-red fluorescent rhodopsin. Proc Natl Acad Sci 111: 13034-13039, 2014.

Packer AM, Russell LE, Dalgleish HWP, Häusser M. Simultaneous all-optical manipulation and recording of neural circuit activity with cellular resolution in vivo. Nat Methods 12: 140-146, 2015.

Park J, Werley CA, Venkatachalam V, Kralj JM, Dib-Hajj SD, Waxman SG, Cohen AE. Screening Fluorescent Voltage Indicators with Spontaneously Spiking HEK Cells. PLOS ONE 8: e85221, 2013.

Perálvarez-Marín A, Lórenz-Fonfría VA, Simón-Vázquez R, Gomariz M, Meseguer I, Querol E, Padrós E. Influence of proline on the thermostability of the active site and membrane arrangement of transmembrane proteins. Biophys J 95: 4384-4395, 2008.

Piatkevich KD, Jung EE, Straub C, Linghu C, Park D, Suk H-J, Hochbaum DR, Goodwin D, Pnevmatikakis E, Pak N, Kawashima T, Yang C-T, Rhoades JL, Shemesh O, Asano S, Yoon Y-G, Freifeld L, Saulnier JL, Riegler C, Engert F, Hughes T, Drobizhev M, Szabo B, Ahrens MB, Flavell SW, Sabatini BL, Boyden ES. A robotic multidimensional directed evolution approach applied to fluorescent voltage reporters. Nat Chem Biol 14: 352, 2018.

Pnevmatikakis EA, Giovannucci A. NoRMCorre: An online algorithm for piecewise rigid motion correction of calcium imaging data. J Neurosci Methods 291: 83-94, 2017.

Pologruto TA, Sabatini BL, Svoboda K. Scanlmage: Flexible software for operating laser scanning microscopes. Biomed Eng OnLine 2: 13, 2003.

St-Pierre F, Marshall JD, Yang Y, Gong Y, Schnitzer MJ, Lin MZ. High-fidelity optical reporting of neuronal electrical activity with an ultrafast fluorescent voltage sensor. Nat Neurosci 17: 884-889, 2014.

Ting JonathanT, Daigle TanyaL, Chen Q, Feng G. Acute Brain Slice Methods for Adult and Aging Animals: Application of Targeted Patch Clamp Analysis and Optogenetics. In: Patch-Clamp Methods and Protocols, edited by Martina M, Taverna S. Springer New York, p. 221-242.

Wagner NL, Greco JA, Ranaghan MJ, Birge RR. Directed evolution of bacteriorhodopsin for applications in bioelectronics. J R Soc Interface 10: 20130197, 2013. 
Wardill TJ, Chen T-W, Schreiter ER, Hasseman JP, Tsegaye G, Fosque BF, Behnam R, Shields BC, Ramirez M, Kimmel BE, Kerr RA, Jayaraman V, Looger LL, Svoboda K, Kim DS. A Neuron-Based Screening Platform for Optimizing Genetically-Encoded Calcium Indicators. PLOS ONE 8: e77728, 2013.

Xu Y, Zou P, Cohen AE. Voltage imaging with genetically encoded indicators. Curr Opin Chem Biol 39: 1-10, 2017.

Yardeni T, Eckhaus M, Morris HD, Huizing M, Hoogstraten-Miller S. Retro-orbital injections in mice. Lab Anim 40: 155-160, 2011.

Zheng Q, Ayala AX, Chung I, Weigel AV, Ranjan A, Falco N, Grimm JB, Tkachuk AN, Wu C, LippincottSchwartz J, Singer RH, Lavis LD. Rational Design of Fluorogenic and Spontaneously Blinking Labels for Super-Resolution Imaging. ACS Cent Sci 5: 1602-1613, 2019.

Zhou M, Liang F, Xiong XR, Li L, Li H, Xiao Z, Tao HW, Zhang LI. Scaling down of balanced excitation and inhibition by active behavioral states in auditory cortex. Nat Neurosci 17: 841-850, 2014.

Zou P, Zhao Y, Douglass AD, Hochbaum DR, Brinks D, Werley CA, Harrison DJ, Campbell RE, Cohen AE. Bright and fast multicoloured voltage reporters via electrochromic FRET. Nat Commun 5: 4625, 2014. 
bioRxiv preprint doi: https://doi.org/10.1101/2021.11.09.467909; this version posted November 11, 2021. The copyright holder for this preprint (which was not certified by peer review) is the author/funder, who has granted bioRxiv a license to display the preprint in perpetuity. It is made available under aCC-BY-NC-ND 4.0 International license.

\section{Author contributions}

We use the CRediT taxonomy to clarify author contributions.

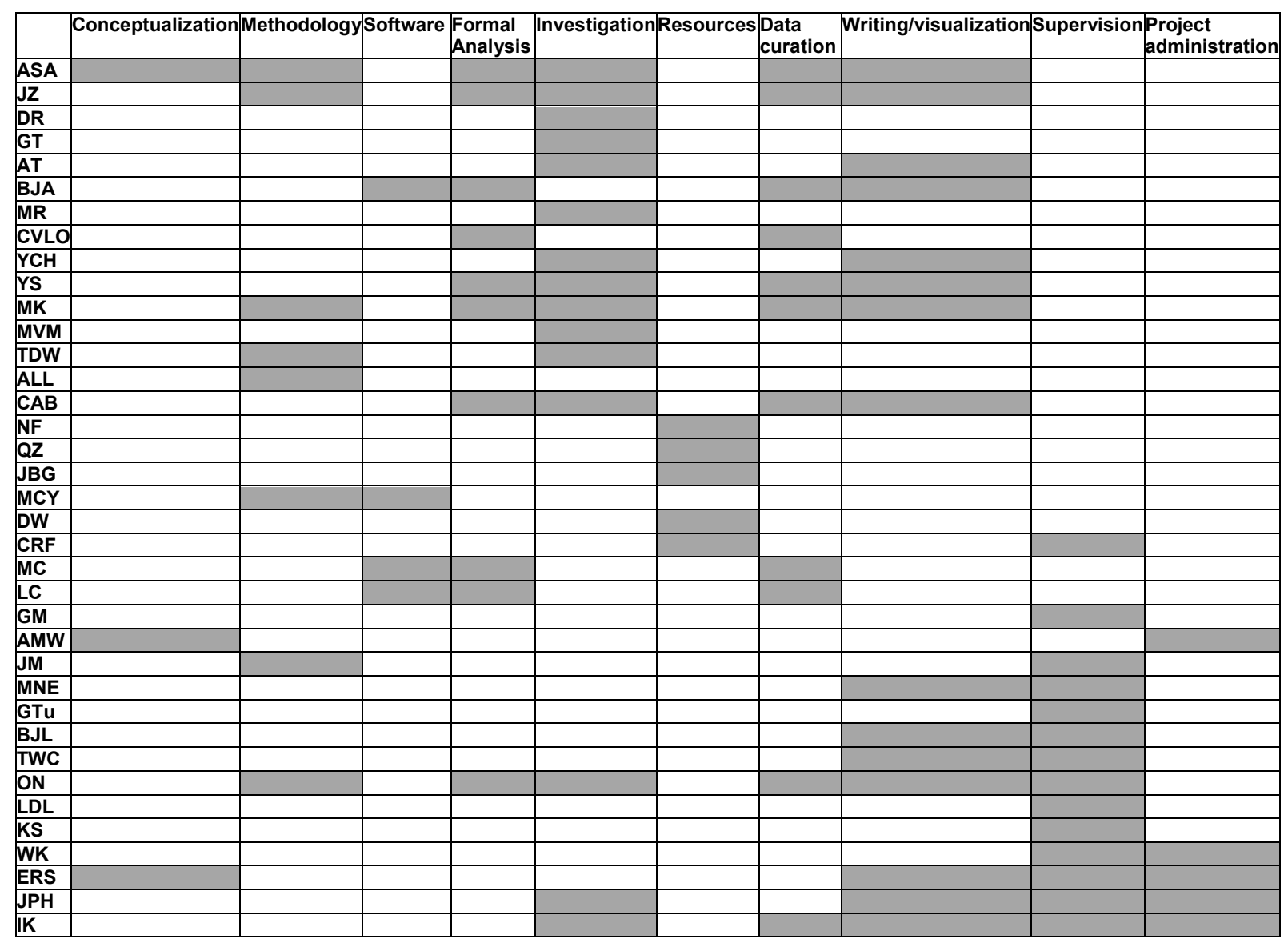

\section{Acknowledgements}

We thank the Janelia cell culture, viviarium, jET, Molecular biology, and Virus Production teams for their assistance. We thank S. Picard, K. Aswath, S. Shrestha for help with DeepSeq. ON, MR, CVLO acknowledge funding from Charles University Grant - PRIMUS/19/MED/003.

\section{Competing interests}

A.S.A., L.D.L., and E.R.S. have filed for a patent on chemigenetic voltage indicators. IK and CRF are coinventors on a patent describing pipette cleaning that is licensed by Sensapex. MC and LC have performed consulting services for Sensapex. 
bioRxiv preprint doi: https://doi.org/10.1101/2021.11.09.467909; this version posted November 11,2021 . The copyright holder for this preprint (which was not certified by peer review) is the author/funder, who has granted bioRxiv a license to display the preprint in perpetuity. It is made available under aCC-BY-NC-ND 4.0 International license.

\section{Figures}

a

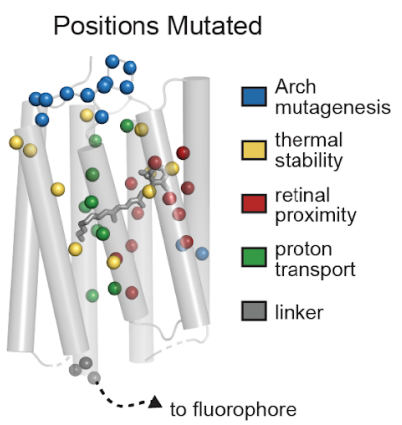

C

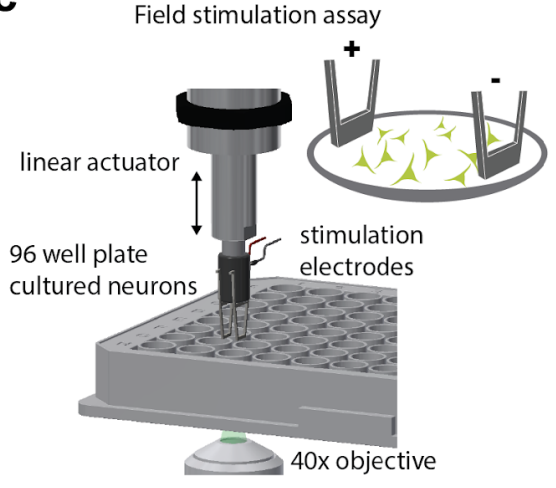

b

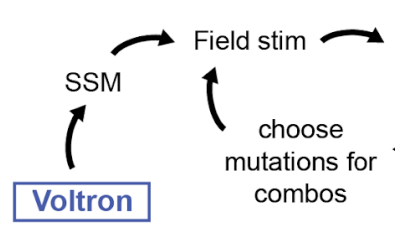

Automated

patch-clamp of

)$_{\substack{1 \text { st } \\ \text { round } \\ \text { hits }}}^{\substack{2^{\text {nd }} \\ \text { round }}}$

Confirm best hits from

SSM and combo

mutations

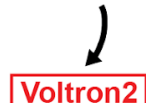

e

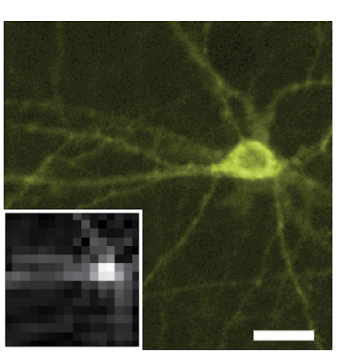

field stimulation

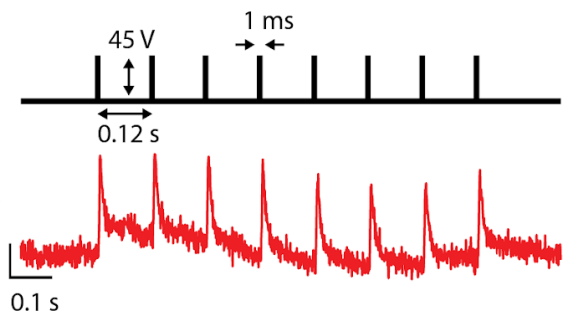

g

$\mathbf{f}$

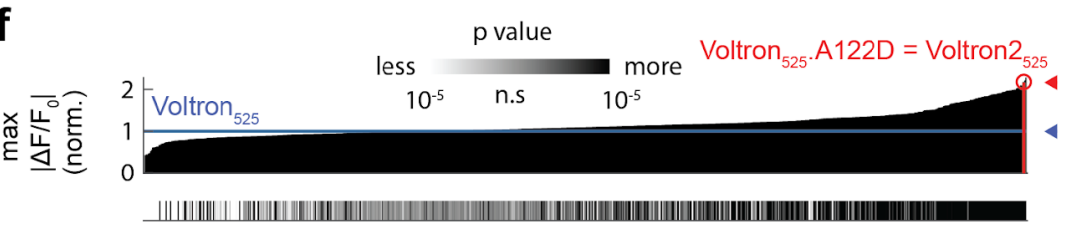

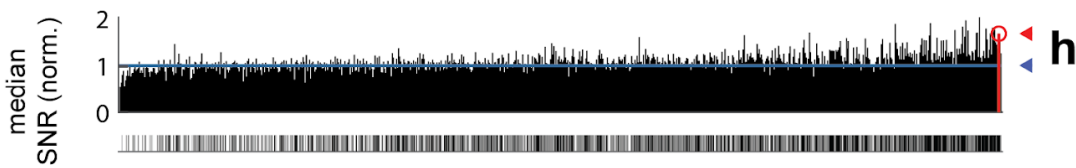

空
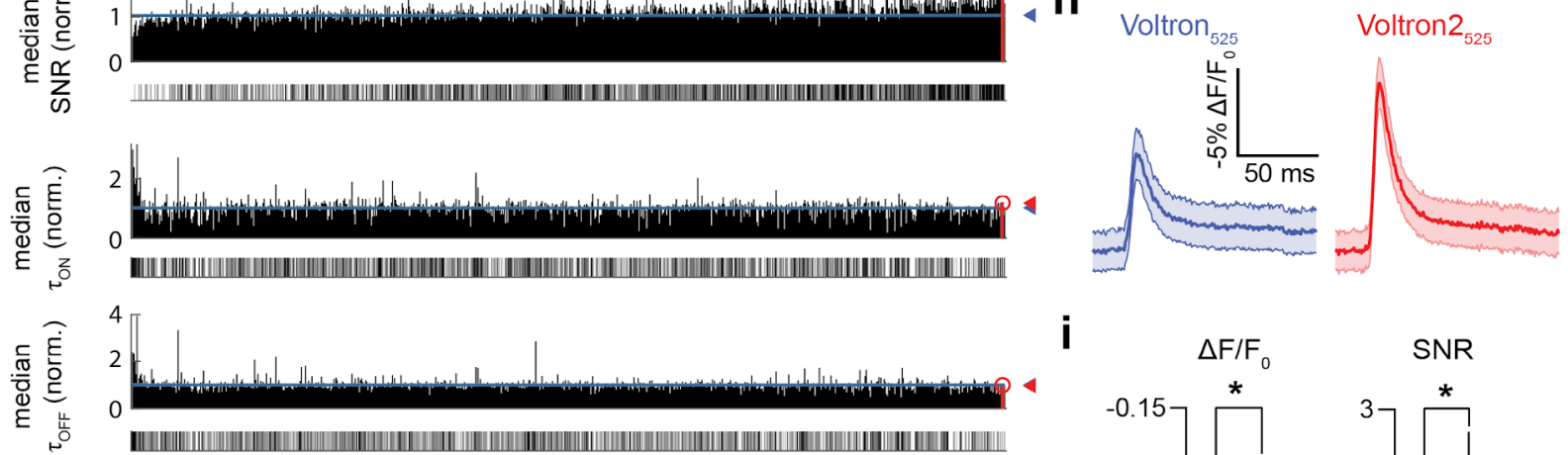

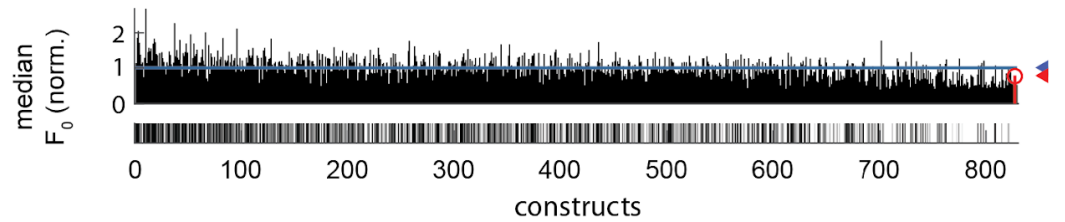

\section{i}
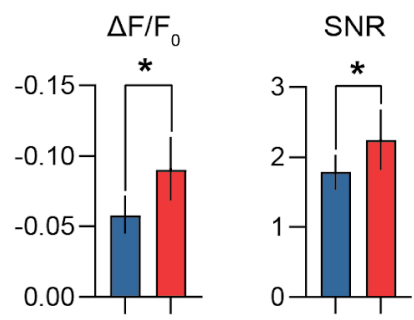

Figure 1: Mutagenesis and screening of Voltron in cultured neurons. a. Residues targeted for SSM in the Ace $2 \mathrm{~N}$ rhodopsin domain of Voltron, colored by the rationale for targeting them. $\mathrm{b}$. Mutagenesis and screening workflow. c. Diagram of field stimulation assay performed in 96-well plates. d. Representative image of neuron from the screen expressing Voltron2 labeled with $\mathrm{JF}_{525}$, Voltron2 ${ }_{525}$. Inset shows representative frame during 
bioRxiv preprint doi: https://doi.org/10.1101/2021.11.09.467909; this version posted November 11,2021 . The copyright holder for this preprint (which was not certified by peer review) is the author/funder, who has granted bioRxiv a license to display the preprint in perpetuity. It is made available under aCC-BY-NC-ND 4.0 International license.

fast $(1,497 \mathrm{~Hz})$ stream acquisition. Scale bar: $10 \mu \mathrm{m}$. e. Field stimulation parameters (top, black) and acquired fluorescence response of the neuron shown in d (bottom, red). All imaging in the screen was performed at a light density of $1.14 \mathrm{~mW} / \mathrm{mm}^{2}$ measured in the image plane. f. Results of single-mutation Voltron $525 \mathrm{screen}_{\text {, }}$ ranked by maximum $\left|\Delta F / F_{0}\right|$ for each variant, normalized to in-plate Voltron 525 controls (top). Color-coded $p$ values are shown below each plot, indicating significant difference compared to in-plate controls. g. Mutated residues colored by the maximum increase in $\left|\Delta F / F_{0}\right|$ achieved in that position. Top three mutations are labeled. h. Representative traces (mean \pm s.e.m.) from a single plate containing Voltron 525 (8 wells) and Voltron2525 (8 wells). i. Single AP $\Delta F / F_{0}$ (Voltron $_{525},-.059 \pm 0.001, n=338$ wells; Voltron2525: $-0.090 \pm 0.002$, $\mathrm{n}=130$ wells; $\mathrm{P}<0.0001$, Mann-Whitney $\mathrm{U}$ test) and SNR of Voltron2 525 and SNR (Voltron $525: 1.80 \pm 0.013$; Voltron2525: $2.24 \pm 0.040 ; \mathrm{P}<0.0001$, Mann-Whitney $U$ test). 
bioRxiv preprint doi: https://doi.org/10.1101/2021.11.09.467909. this version posted November 11 2021. The copyright holder for this preprint (which was not certified by peer review) is the author/funder, who has granted bioRxiv a license to display the preprint in perpetuity. It is made available under aCC-BY-NC-ND 4.0 International license.

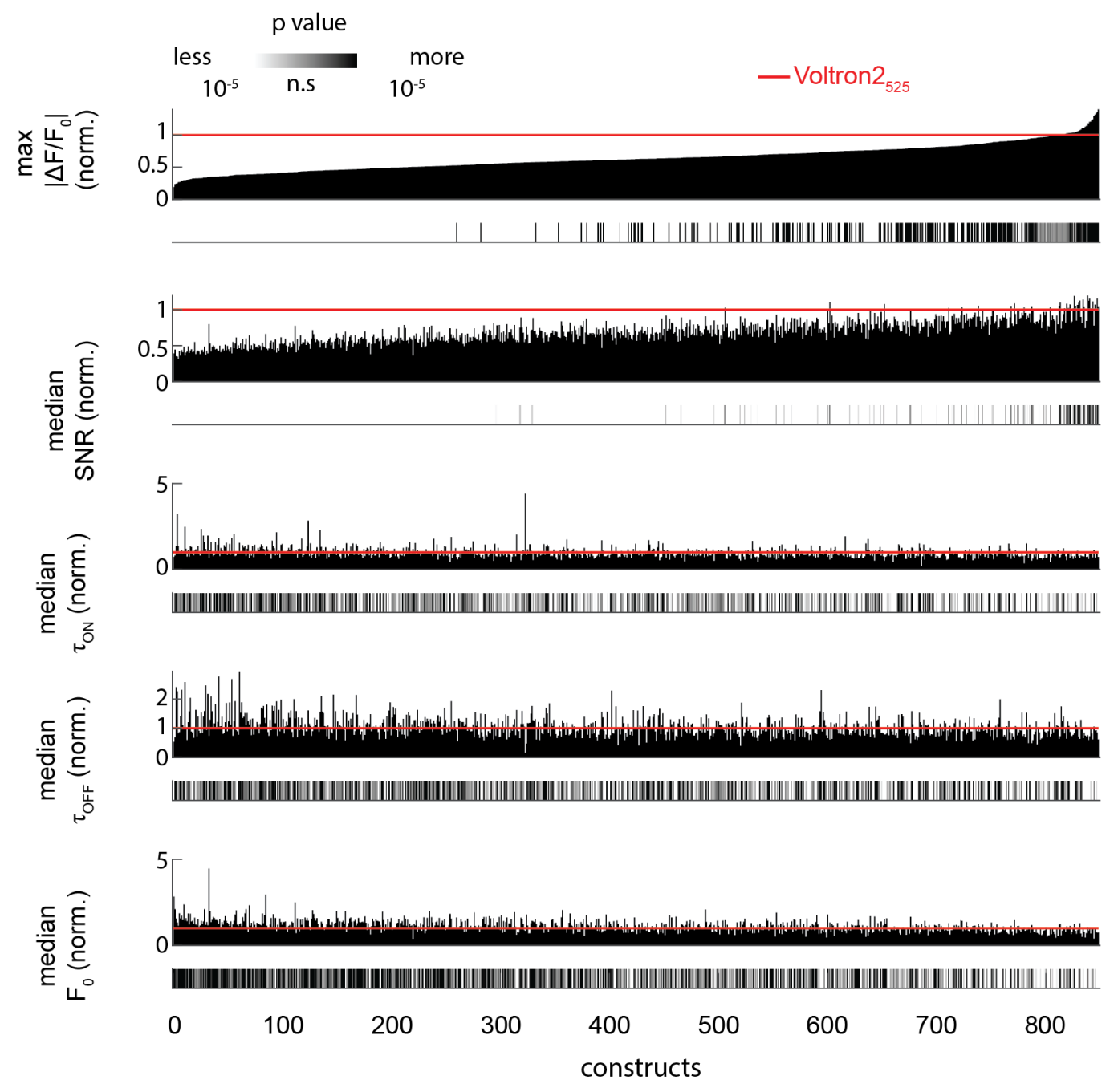

Supplementary Figure 1: Results of combo mutation screen, ranked by maximum $\left|\Delta F / F_{0}\right|$ for each variant, normalized to in-plate Voltron $2_{525}$ controls. $P$ values indicate significant difference compared to Voltron 525 inplate controls. 
bioRxiv preprint doi: https://doi.org/10.1101/2021.11.09.467909; this version posted November 11, 2021. The copyright holder for this preprint (which was not certified by peer review) is the author/funder, who has granted bioRxiv a license to display the preprint in perpetuity. It is made available under aCC-BY-NC-ND 4.0 International license.

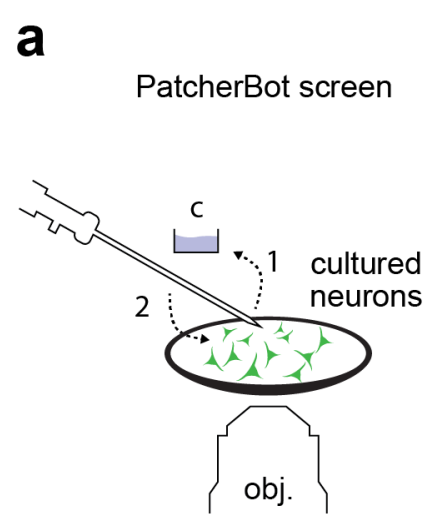

d

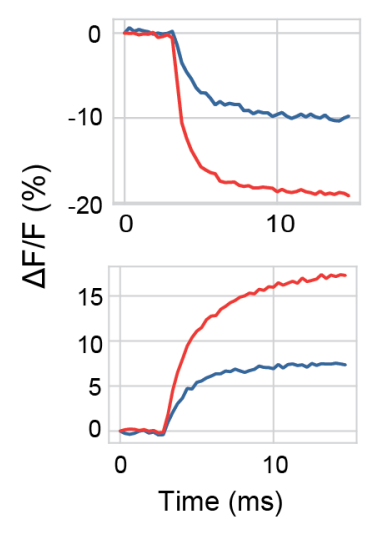

f
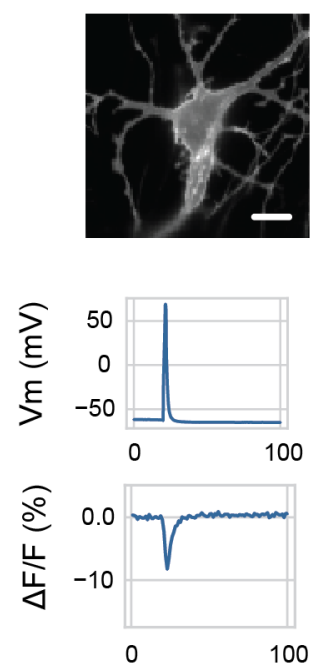

b

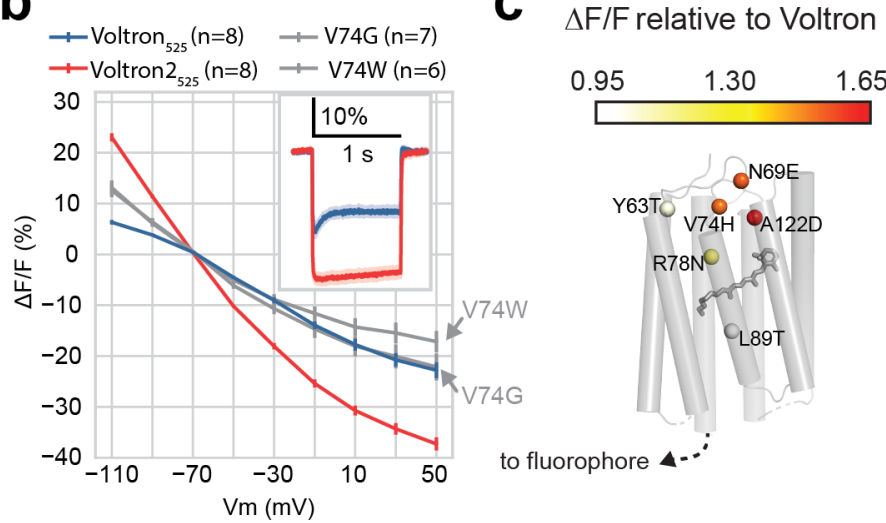

e

onset kinetics
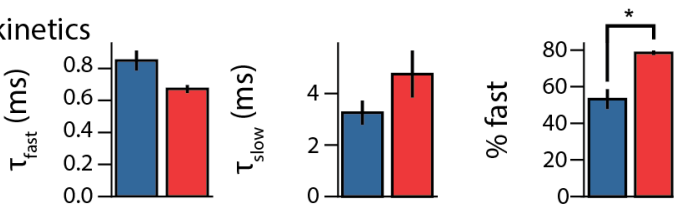

decay kinetics
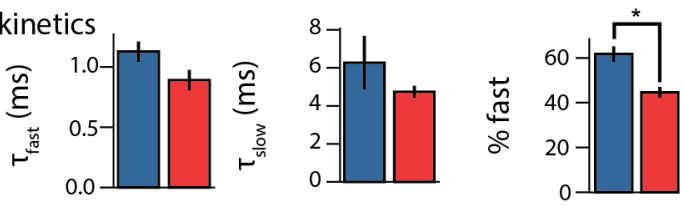

g
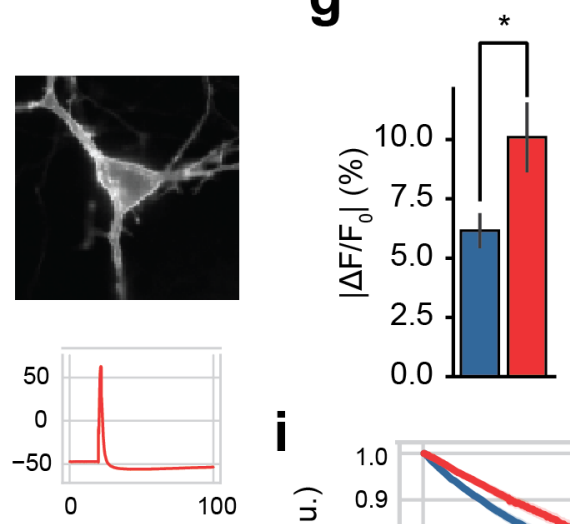

h
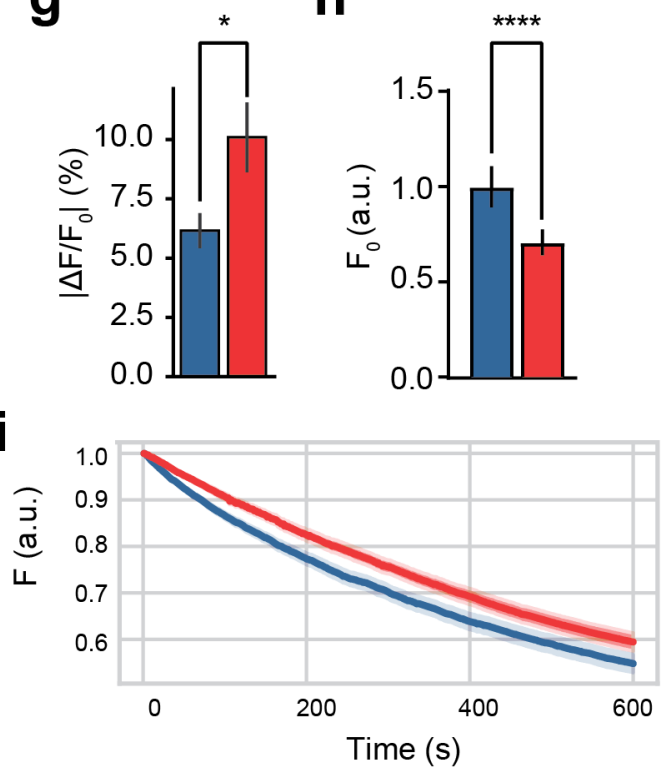

Figure 2: Automated patch-clamp screening and Voltron2 characterization in cultured neurons. a. Fully automated uM workstation screening platform, based on PatcherBot. The pipette cleaning procedure is shown where a used pipette is dipped into a reservoir of cleaning solution (step 1, "c") and back to the neuronal culture for a subsequent patch-clamp attempt without the need for replacing the pipette (step 2). b. Peak fluorescence response to voltage steps from $-70 \mathrm{mV}$ of Voltron ${ }_{525}$, Voltron2 525 and the top two variants from the field stimulation assay (Voltron2 ${ }_{525}$ Vs. Voltron 525 : $P=0.012$; Voltron2 ${ }_{525}$ vs. Voltron ${ }_{525}$. V74G: $P=0.015$; Voltron ${ }_{525}$ Vs. Voltron $525 . V 74 W$ : $P=0.0003$, one-way ANOVA followed by Dunnett's post-hoc test). Inset: Voltron $_{525}$ and Voltron2 ${ }_{525}$ fluorescence traces (mean \pm s.e.m.) in response to -70 to $+30 \mathrm{mV}$ voltage steps. c. Mutated residues from $1^{\text {st }}$ screening round (single sites) colored by the maximum $\Delta F / F_{0}$ response to $100 \mathrm{mV}(-$ 
bioRxiv preprint doi: https://doi.org/10.1101/2021.11.09.467909. this version posted November 11,2021 . The copyright holder for this preprint (which was not certified by peer review) is the author/funder, who has granted bioRxiv a license to display the preprint in perpetuity. It is made available under aCC-BY-NC-ND 4.0 International license.

70 to $+30 \mathrm{mV}$ ) voltage steps, measured with the uM workstation. Top mutations at each position are labeled. $\mathrm{d}$. Representative onset (top) and decay (bottom) fluorescence kinetics of Voltron 525 and Voltron2 ${ }_{525}$ in response to $\mathrm{a}+100 \mathrm{mV}$ voltage step from $-70 \mathrm{mV}$. e. Onset and decay kinetics. Onset kinetics: * $\mathrm{P}=0.03$, Mann-Whitney $U$ test. Decay kinetics: * $P=0.03$, Mann-Whitney $U$ test. f. Representative fluorescence responses to single evoked APs in current clamp. Scale bar: $10 \mu \mathrm{m}$. g. $\Delta F / F_{0}$ in response to single AP stimulation in current clamp mode ( ${ }^{*} P=0.03$, Student's unpaired $t$ test). $h$. Normalized resting fluorescence relative to $m T a g B F P 2$ fused to the $C$ terminus ( ${ }^{* * *} P<0.0001$; Voltron ${ }_{525}: n=105$ cells, Voltron2 ${ }_{525}: n=115$ cells). i. Photobleaching comparison of Voltron 525 and Voltron2 525 over 10 mins. 
bioRxiv preprint doi: https://doi.org/10.1101/2021.11.09.467909; this version posted November 11,2021 . The copyright holder for this preprint (which was not certified by peer review) is the author/funder, who has granted bioRxiv a license to display the preprint in perpetuity. It is made available under aCC-BY-NC-ND 4.0 International license.

a

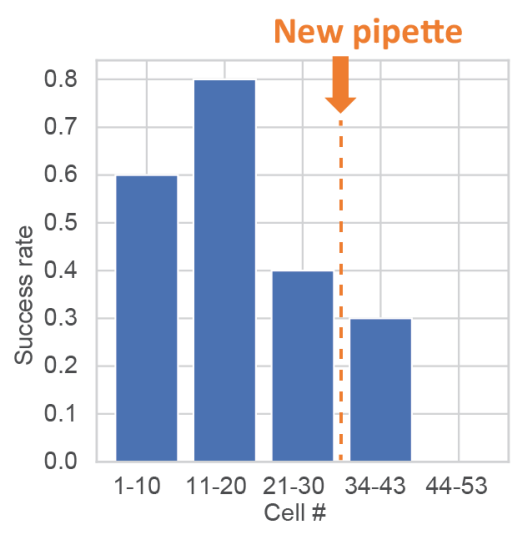

C

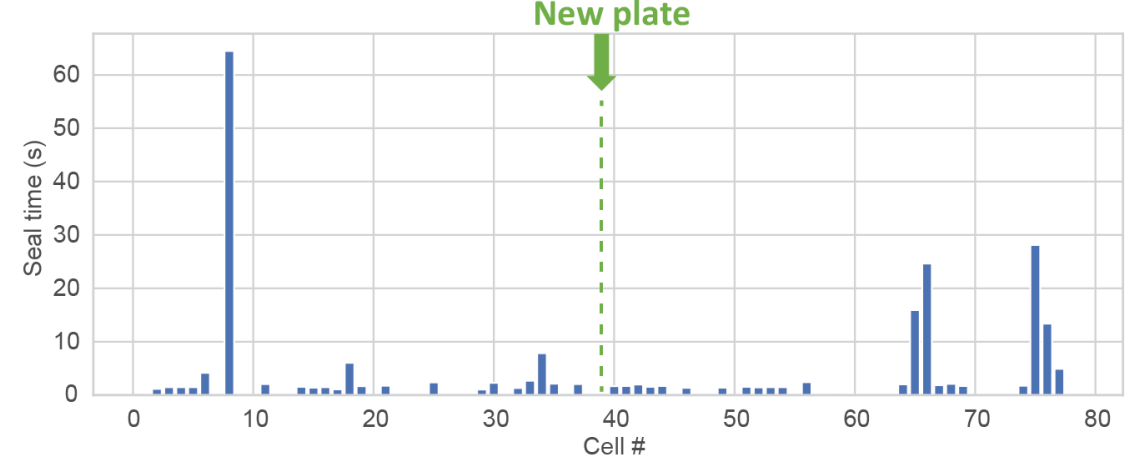

b

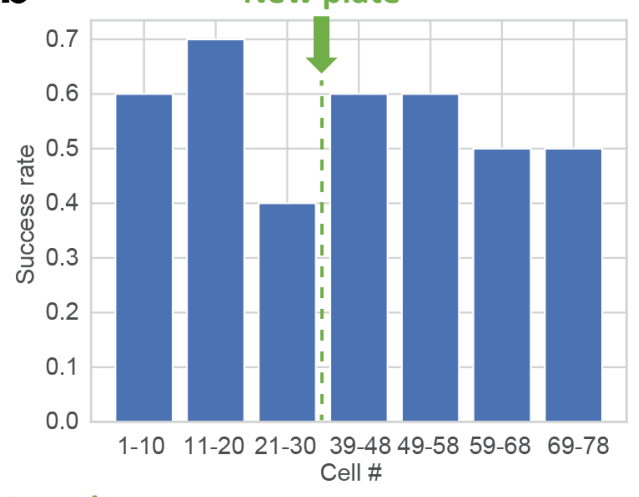

New plate

Supplementary Figure 2: Pipette cleaning with the uM Workstation. a. Whole-cell recording success rate with pipette cleaning after every recording. The pipette was replaced after 30 recordings, but the success rate did not improve. b. Whole-cell recording success rate with a single reused pipette. A new plate of neurons was used after the $30^{\text {th }}$ cell, causing the success rate to improve. c. Time to form a gigaseal over multiple cells using a single pipette. A blank entry indicates that the gigaseal was unsuccessful. A new plate of neurons was used after the $38^{\text {th }}$ cell. 
bioRxiv preprint doi: https://doi.org/10.1101/2021.11.09.467909: this version posted November 11, 2021. The copyright holder for this preprint (which was not certified by peer review) is the author/funder, who has granted bioRxiv a license to display the preprint in perpetuity. It is made available under aCC-BY-NC-ND 4.0 International license.

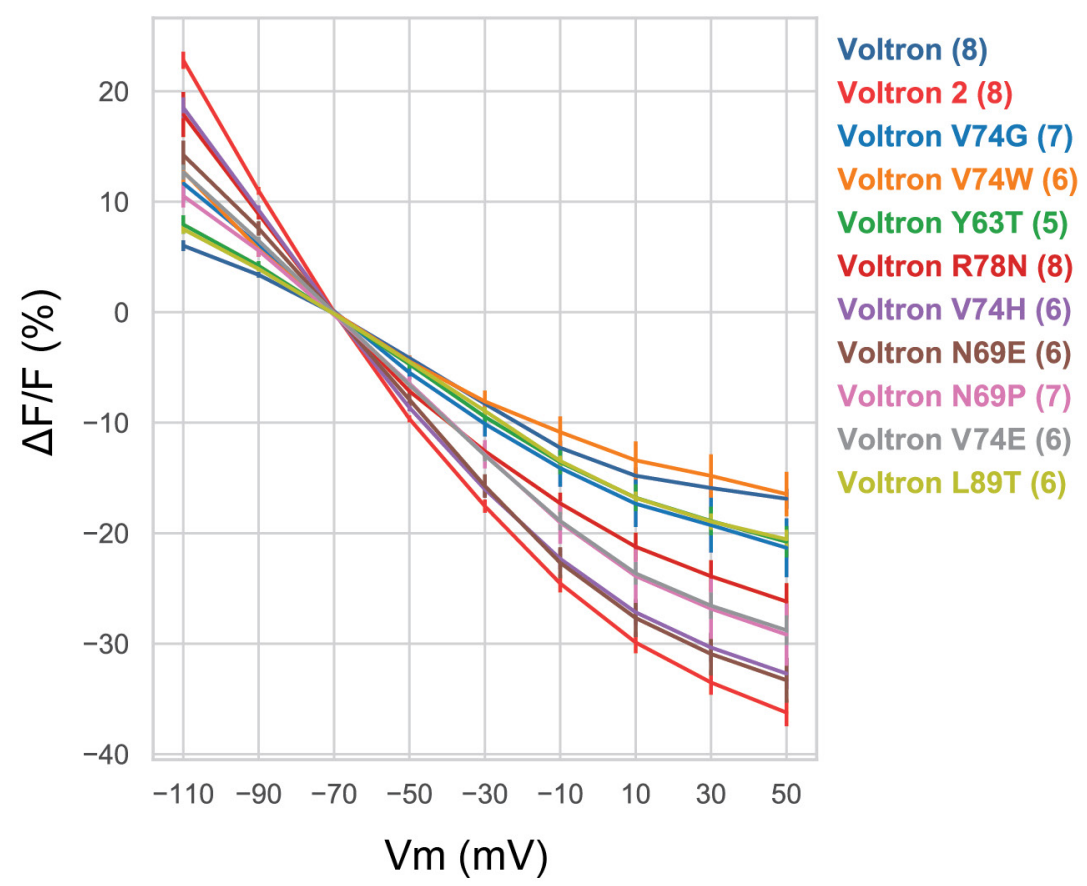

Supplementary Figure 3: Peak fluorescence response to voltage steps from -70 $\mathrm{mV}$ of the top variants from the field stimulation assay, with Voltron and Voltron2 traces (reproduced from Fig. 2) superimposed for reference. Number in parentheses indicates the number of neurons assayed. All sensor mutants were conjugated to $\mathrm{JF}_{525}$ dyes for these experiments (Voltron ${ }_{525}$ ). 
bioRxiv preprint doi: https://doi.org/10.1101/2021.11.09.467909; this version posted November 11,2021 . The copyright holder for this preprint (which was not certified by peer review) is the author/funder, who has granted bioRxiv a license to display the preprint in perpetuity. It is made available under aCC-BY-NC-ND 4.0 International license.

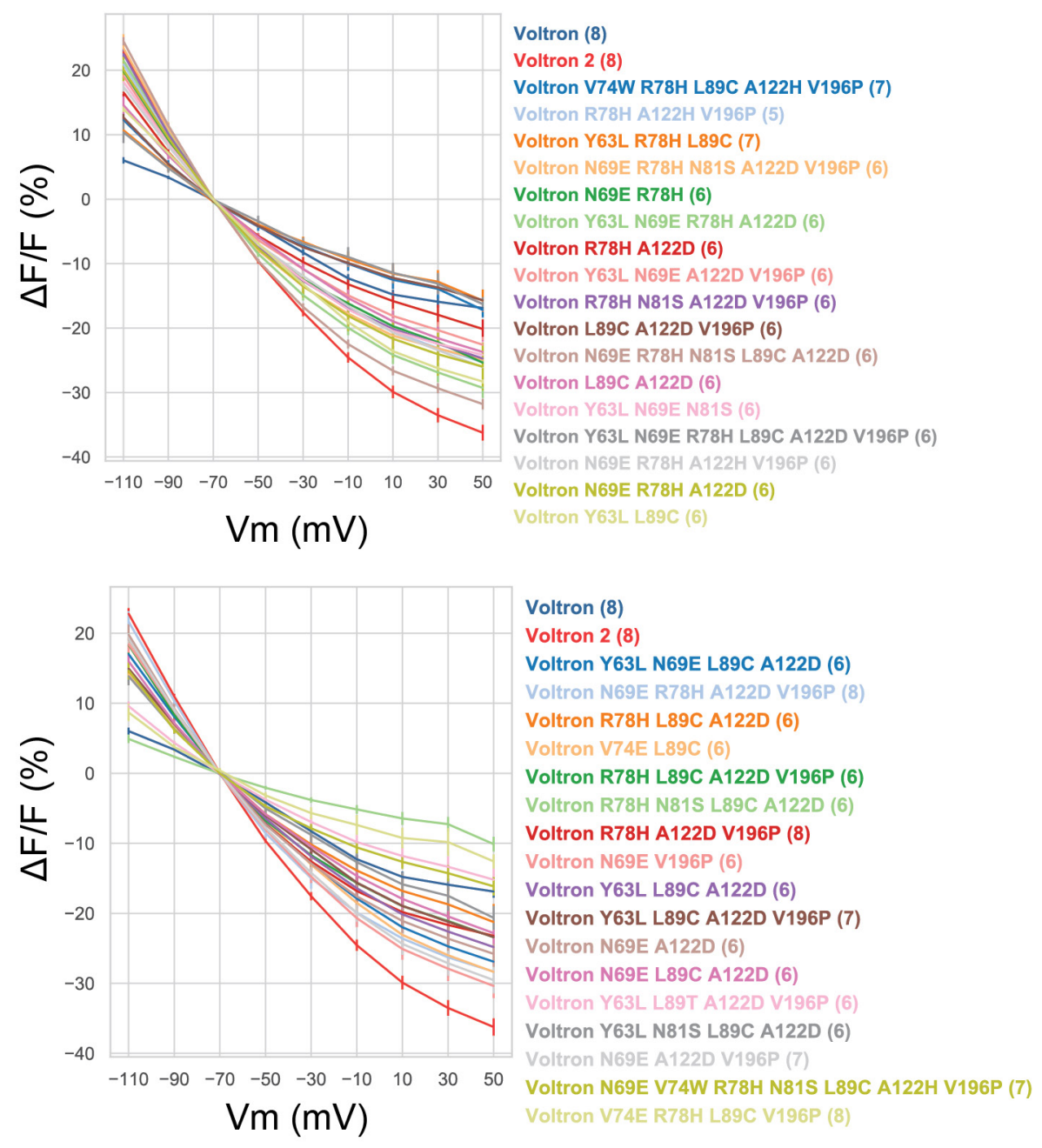

Supplementary Figure 4: Peak fluorescence response to voltage steps of Voltron, Voltron2 (reproduced from Fig. 2) and the top-performing combo variants from the field stimulation assay. For clarity, variants are divided into two panels. Number in parentheses indicates the number of neurons assayed. All sensor mutants were conjugated to $\mathrm{JF}_{525}$ dyes for these experiments (Voltron ${ }_{525}$ ). 
bioRxiv preprint doi: https://doi.org/10.1101/2021.11.09.467909; this version posted November 11, 2021. The copyright holder for this preprint (which was not certified by peer review) is the author/funder, who has granted bioRxiv a license to display the preprint in perpetuity. It is made available under aCC-BY-NC-ND 4.0 International license.

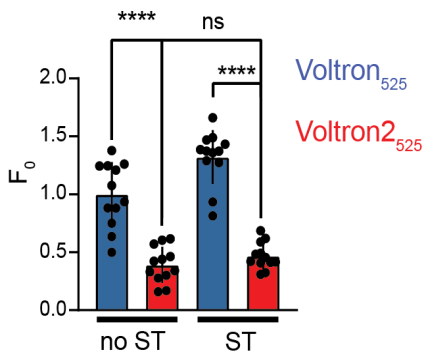

Supplementary Figure 5: Baseline fluorescence of non soma-tagged (no ST) and soma-tagged (ST) Voltron 525 and Voltron2 ${ }_{525}\left(\mathrm{n}=12\right.$ neurons for each, from single transfection; error bars: s.d.; ${ }^{* * *}$ : $\mathrm{P}<0.0001 ; \mathrm{n} . \mathrm{s} .: \mathrm{P}=0.80$; one-way ANOVA followed by Tukey's multiple comparison test). See Supplementary Figs. 6, 7 for cell images. 
bioRxiv preprint doi: https://doi.org/10.1101/2021.11.09.467909; this version posted November 11, 2021. The copyright holder for this preprint (which was not certified by peer review) is the author/funder, who has granted bioRxiv a license to display the preprint in perpetuity. It is made available under aCC-BY-NC-ND 4.0 International license.

a.

\section{Voltron $_{525}$}

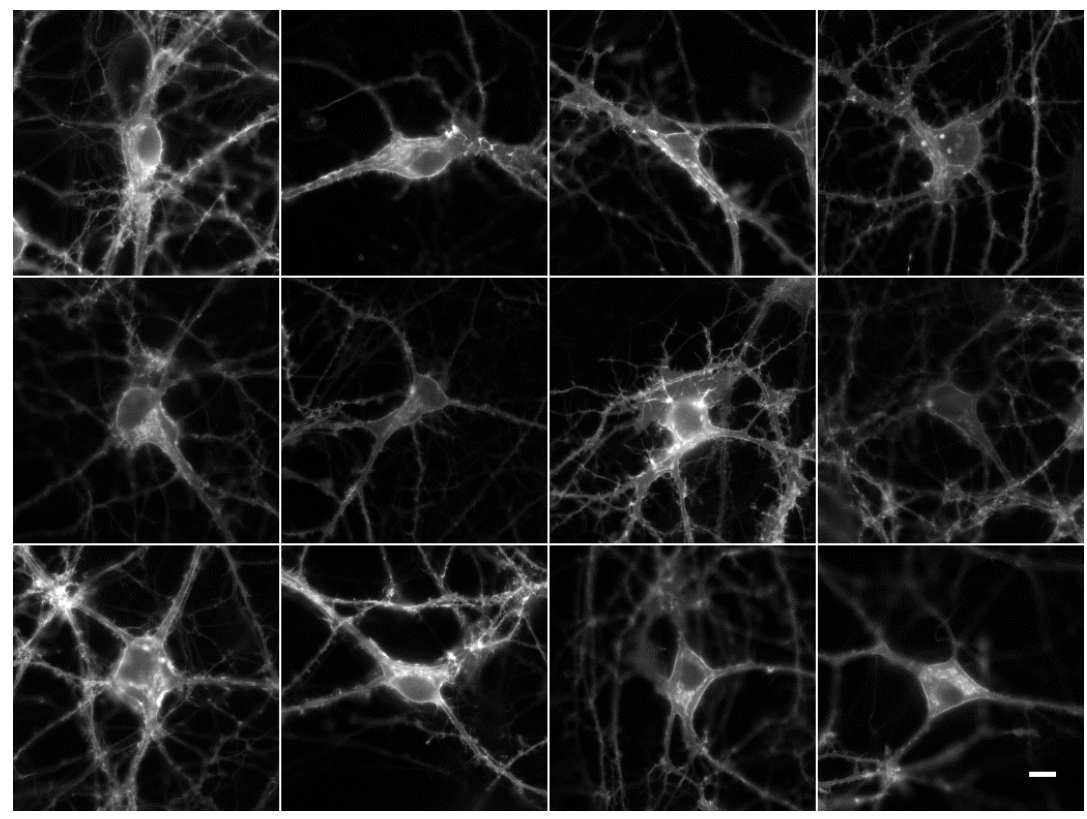

b.

\section{Voltron2 525}

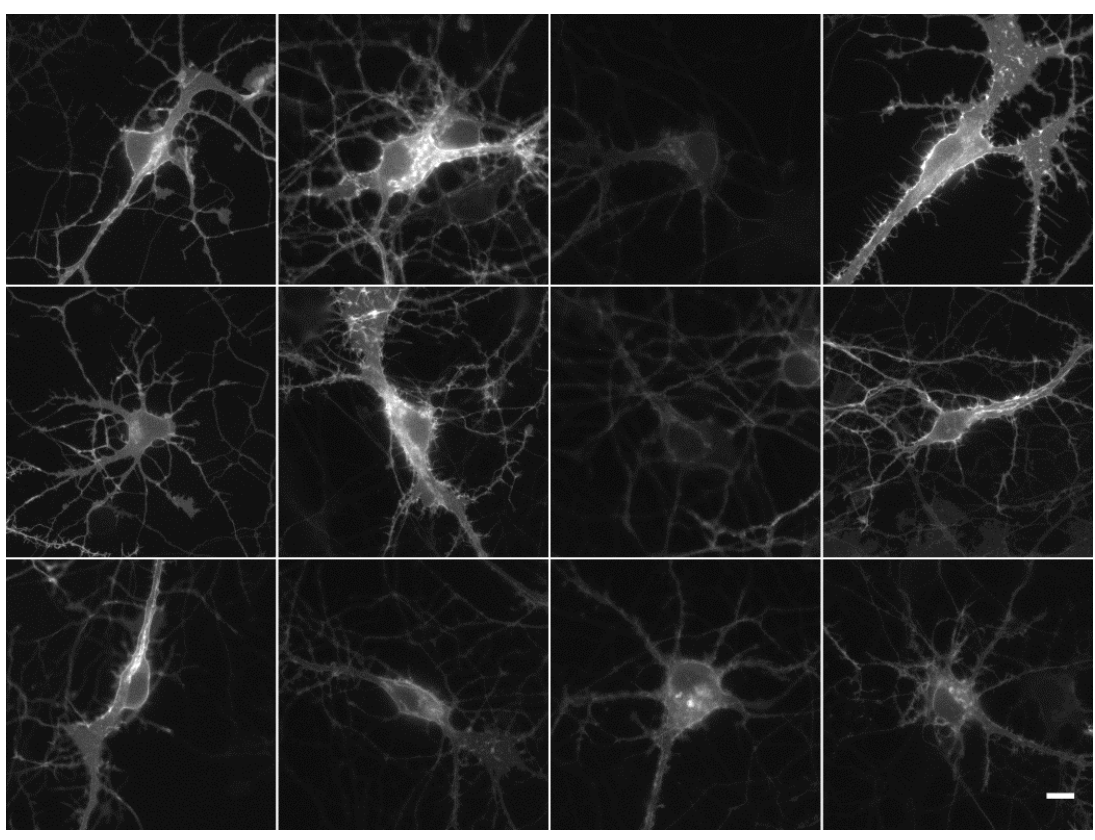

Supplementary Figure 6. Representative fluorescent images of cultured hippocampal neurons expressing (non-soma tagged) Voltron 525 and Voltron2525. Images were taken with $15 \mathrm{~mW} / \mathrm{mm}^{2}$ light power and $30 \mathrm{~ms}$ exposure time. Dynamic ranges of images were rescaled for clarity. For quantitative comparison of fluorescence intensities, see Fig. $2 \mathrm{~h}$. Scale bar: $10 \mu \mathrm{m}$ 
bioRxiv preprint doi: https://doi.org/10.1101/2021.11.09.467909; this version posted November 11, 2021. The copyright holder for this preprint (which was not certified by peer review) is the author/funder, who has granted bioRxiv a license to display the preprint in perpetuity. It is made available under aCC-BY-NC-ND 4.0 International license.

a.

\section{Voltron $_{525}$-ST}

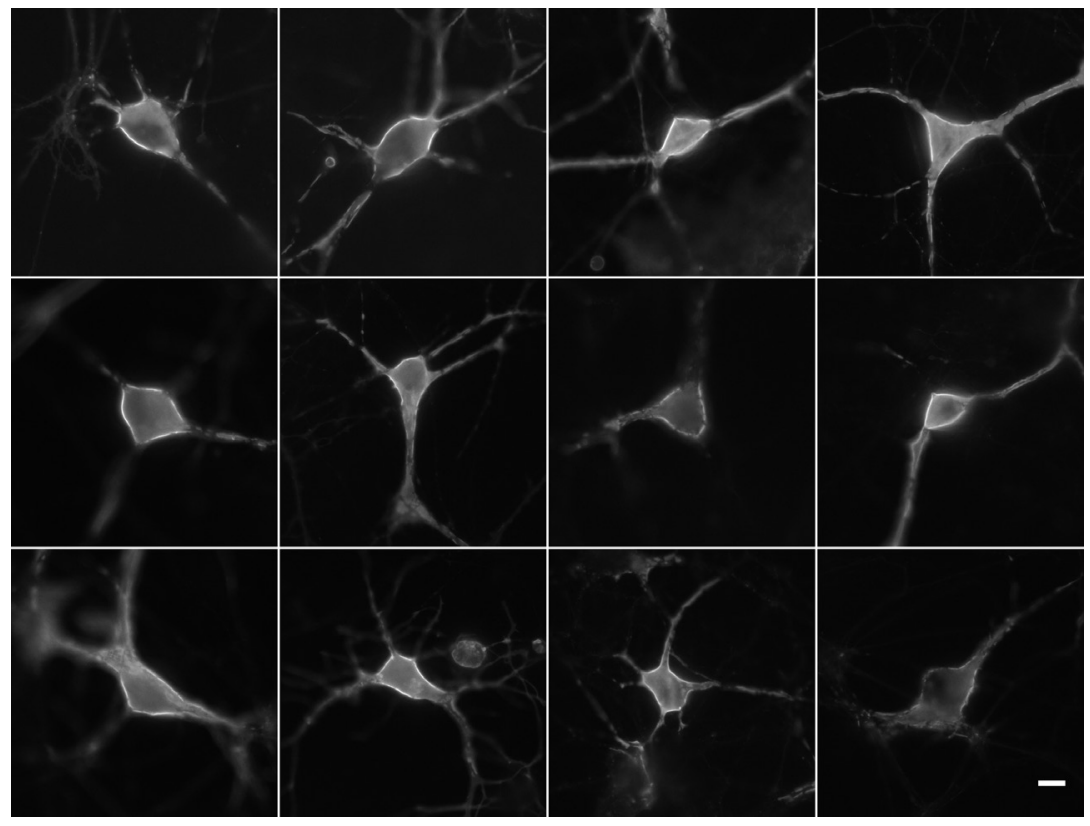

b.

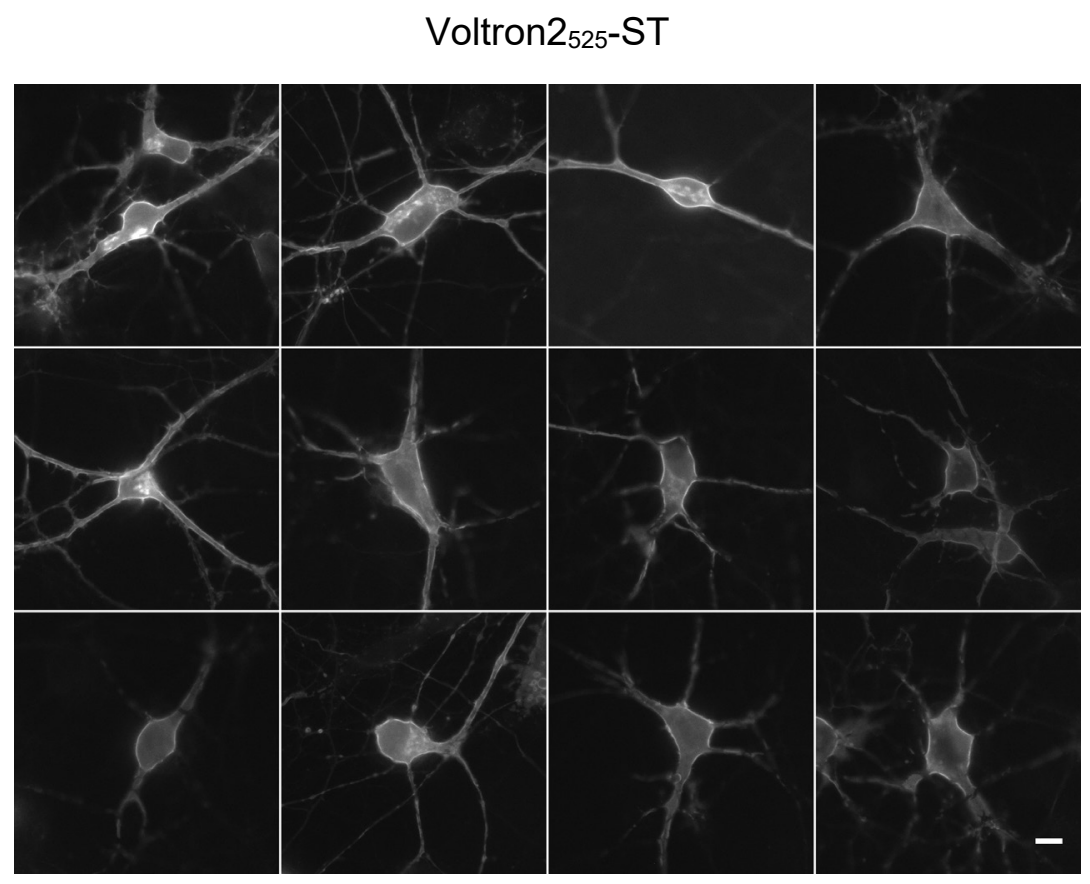

Supplementary Figure 7. Representative fluorescent images of cultured hippocampal neurons expressing soma-tagged Voltron 525 and Voltron $2_{525}$. Images were taken with $15 \mathrm{~mW} / \mathrm{mm}^{2}$ light power and 30 ms exposure time. Dynamic ranges of images were rescaled for clarity. For quantitative comparison of fluorescence intensities, see Fig. 2h. Scale bar: $10 \mu \mathrm{m}$ 
bioRxiv preprint doi: https://doi.org/10.1101/2021.11.09.467909; this version posted November 11, 2021. The copyright holder for this preprint (which was not certified by peer review) is the author/funder, who has granted bioRxiv a license to display the preprint in perpetuity. It is made available under aCC-BY-NC-ND 4.0 International license.

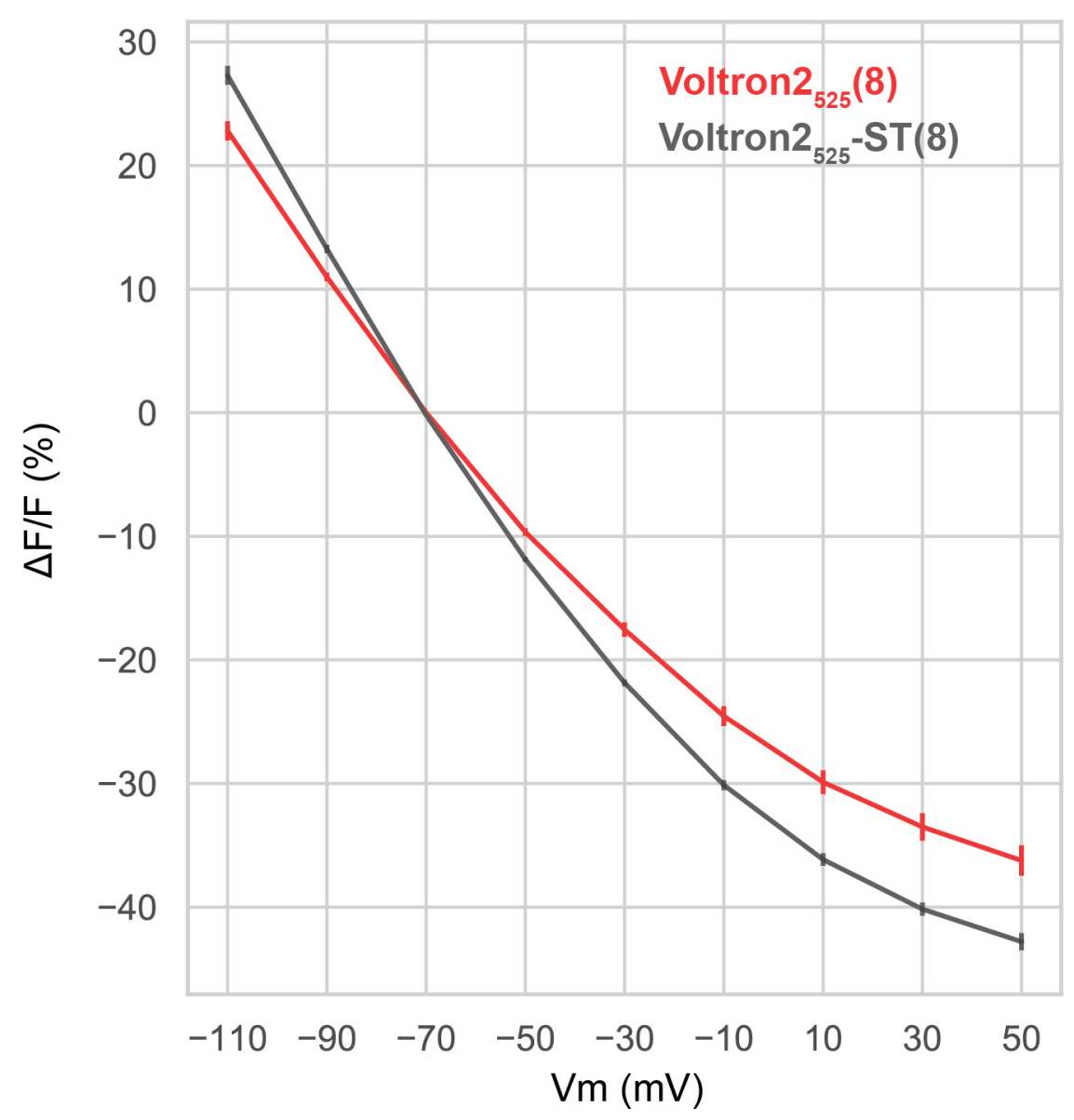

Supplementary Figure 8: Fluorescence response to voltage steps of Voltron2 ${ }_{525}-\mathrm{ST}$, compared to Voltron2 525 (reproduced from Fig. 2). Number in parentheses indicates the number of neurons assayed. 
bioRxiv preprint doi: https://doi.org/10.1101/2021.11.09.467909; this version posted November 11,2021 . The copyright holder for this preprint (which was not certified by peer review) is the author/funder, who has granted bioRxiv a license to display the preprint in perpetuity. It is made available under aCC-BY-NC-ND 4.0 International license.
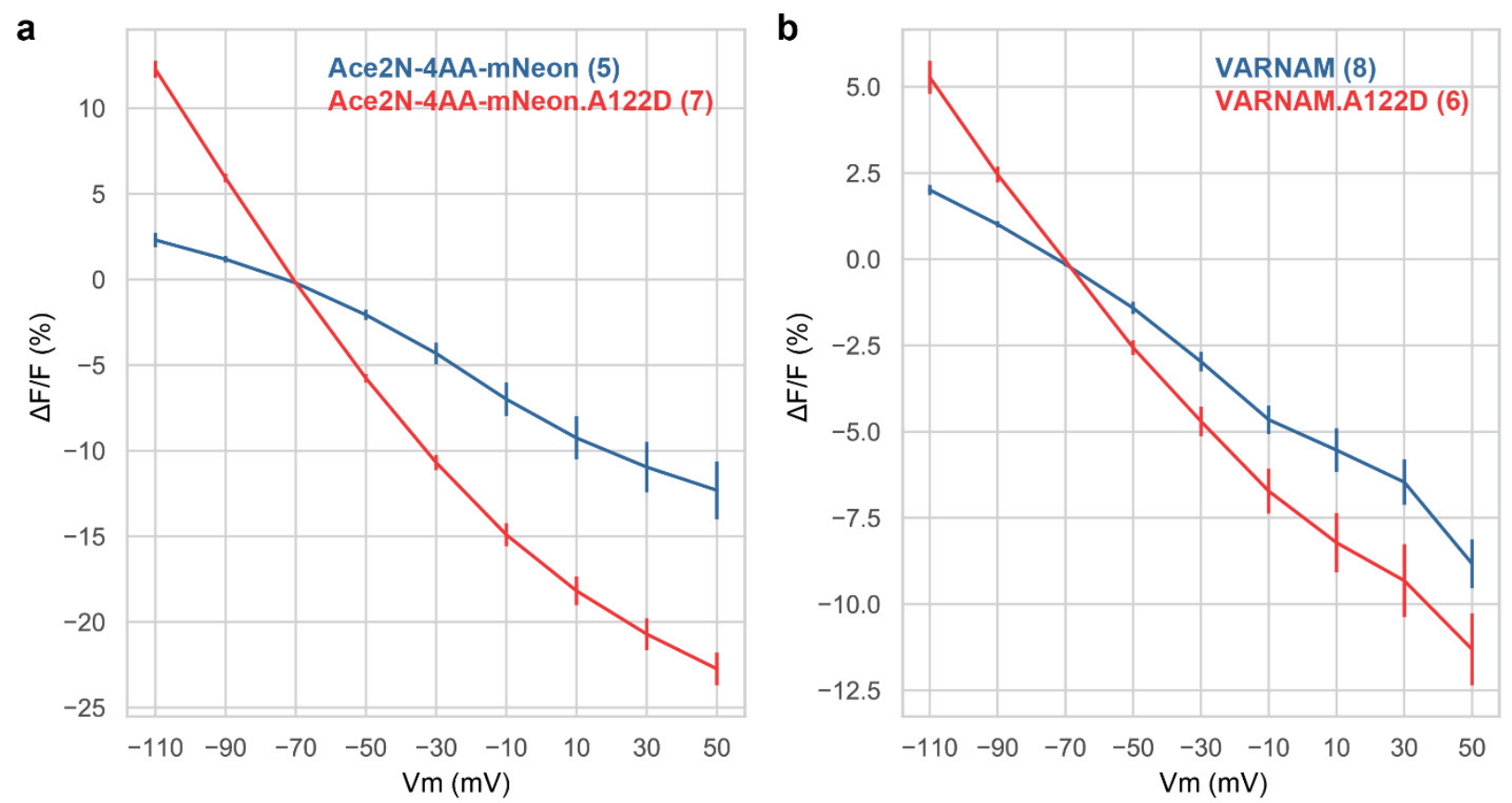

Supplementary Figure 9: Increased voltage sensitivities of Ace-based FRET opsin indicators. a. Peak fluorescence response to voltage steps from $-70 \mathrm{mV}$ of Ace-4AA-mNeon.A122D, with Ace-4AA-mNeon as control. b. Peak fluorescence response to voltage steps from -70 mV of VARNAM.A122D, with VARNAM as control. Number in parentheses indicates the number of neurons assayed. 
bioRxiv preprint doi: $\mathrm{https}$ //doi.org/10.1101/2021.11.09.467909; this version posted November 11, 2021. The copyright holder for this preprint (which was not certified by peer review) is the author/funder, who has granted bioRxiv a license to display the preprint in perpetuity. It is made available under aCC-BY-NC-ND 4.0 International license.

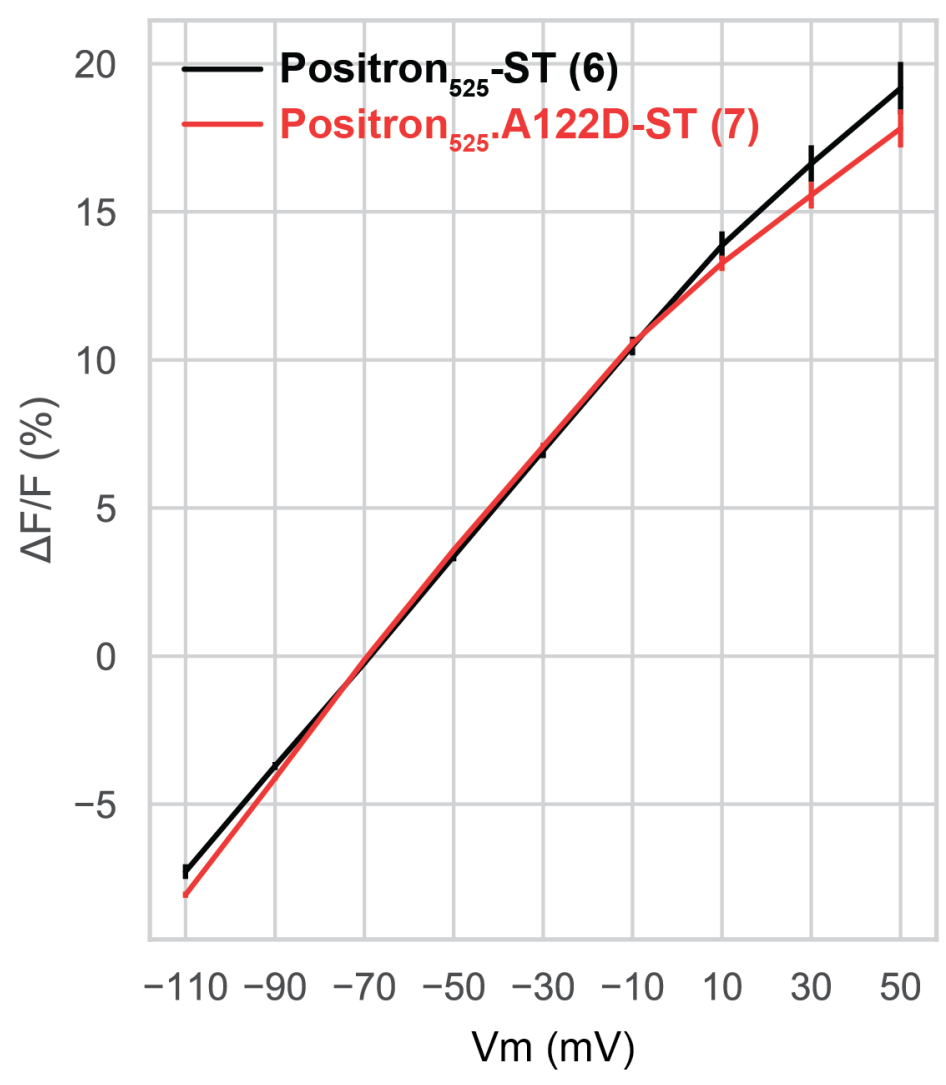

Supplementary Figure 10: Fluorescence response to voltage steps of Positron ${ }_{525}-\mathrm{ST}$, compared to

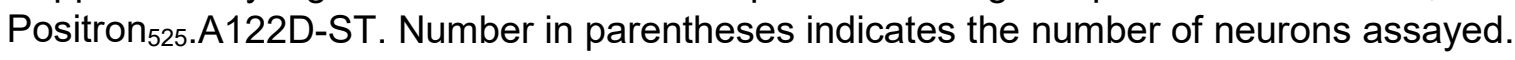


bioRxiv preprint doi: https://doi.org/10.1101/2021.11.09.467909; this version posted November 11,2021 . The copyright holder for this preprint (which was not certified by peer review) is the author/funder, who has granted bioRxiv a license to display the preprint in perpetuity. It is made available under aCC-BY-NC-ND 4.0 International license.

a

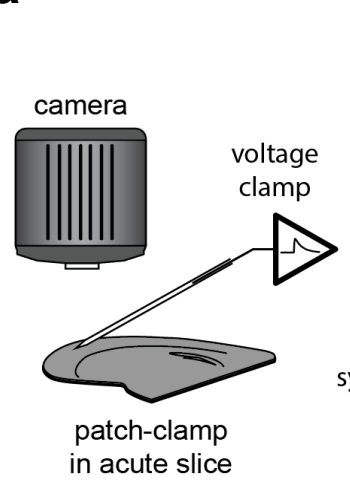

b

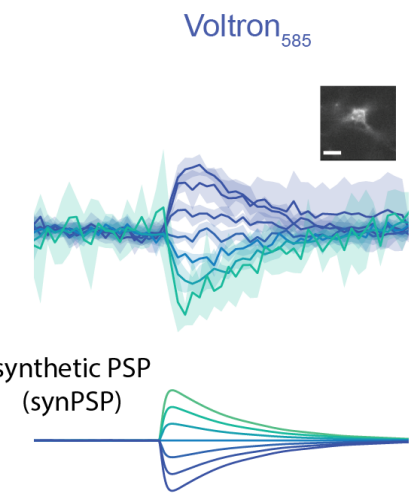

C

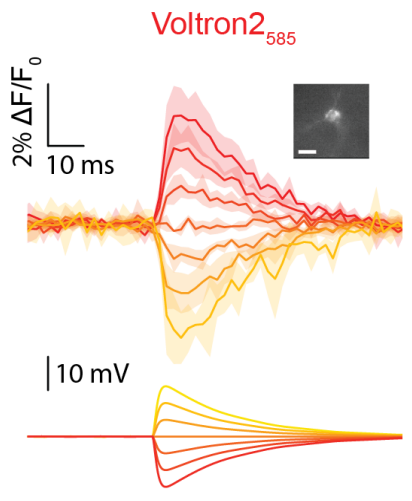

d
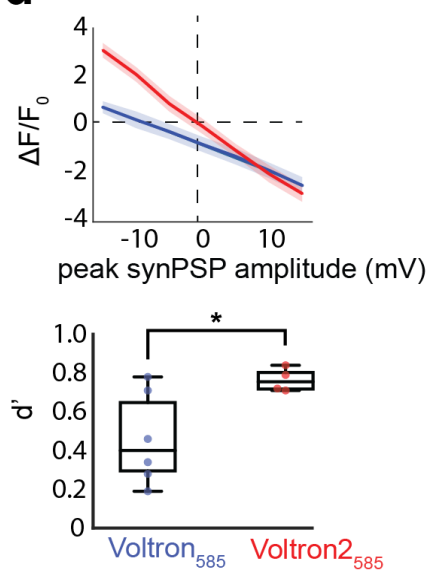

Figure 3: PSP detection using Voltron2 in mouse brain slices. a. Synthetic PSP (synPSP) experimental setup in acute mouse brain slice. b,c. Average \pm SD of percent change in fluorescence over time for Voltron 585 (b; $\mathrm{n}=6$ cells) or Voltron 2585 (c, $n=4$ cells) in response to changes from resting membrane potential of $-15 \mathrm{mV}$ to $+15 \mathrm{mV}$ in $5 \mathrm{mV}$ increments (lower panels), intended to mimic typical inhibitory or excitatory synaptic transmission. A representative cell for each construct is shown in the inset (scale bar $=10 \mu \mathrm{m})$. $\mathrm{d}$. Top: average \pm SD of percent change in fluorescence as a function of the peak amplitude of the synthetic postsynaptic potential (synPSP) applied to the cell. Voltron does not cross the 0,0 point due to photobleaching in the course of the experiment. Bottom: sensitivity index (d') of Voltron2585 is significantly higher than that of Voltron ${ }_{585}$ $(P=0.025$, Welch's t-test). 


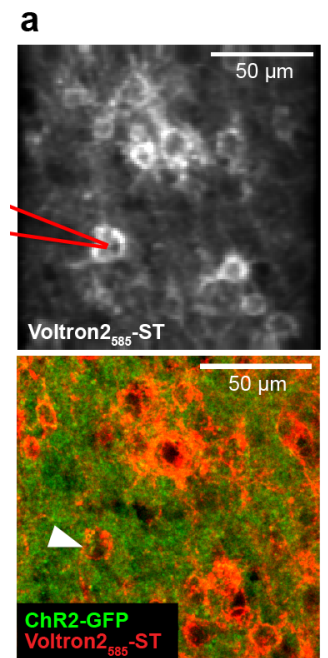

\section{b}

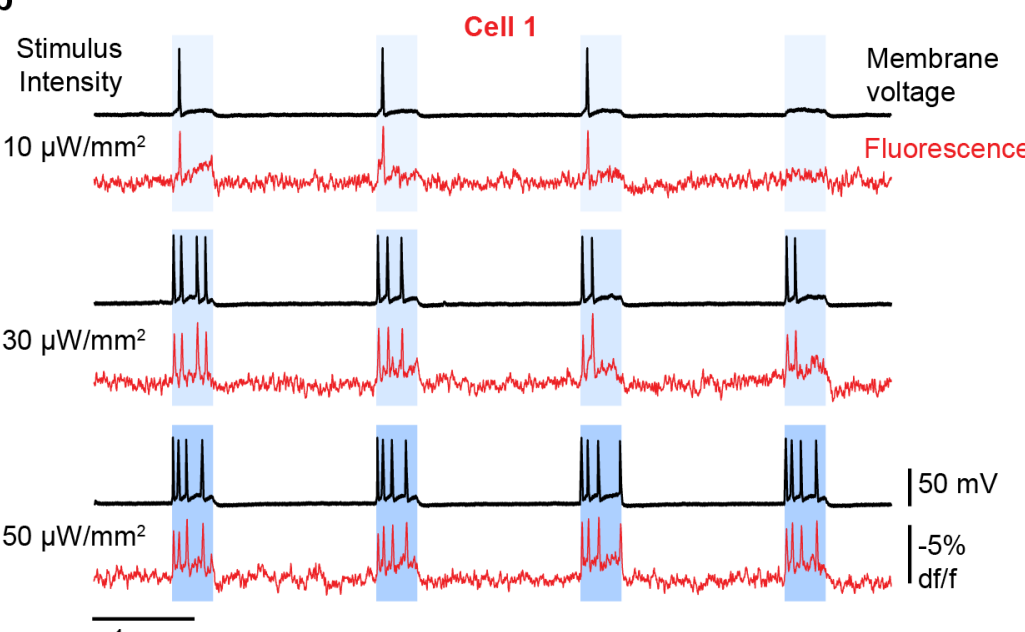

c

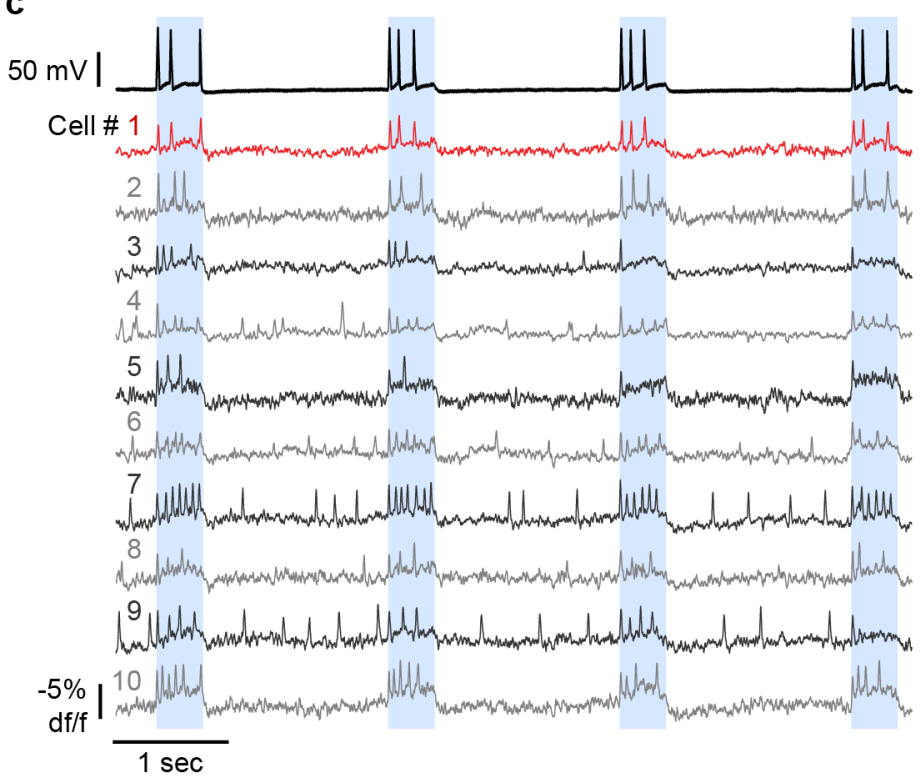

d

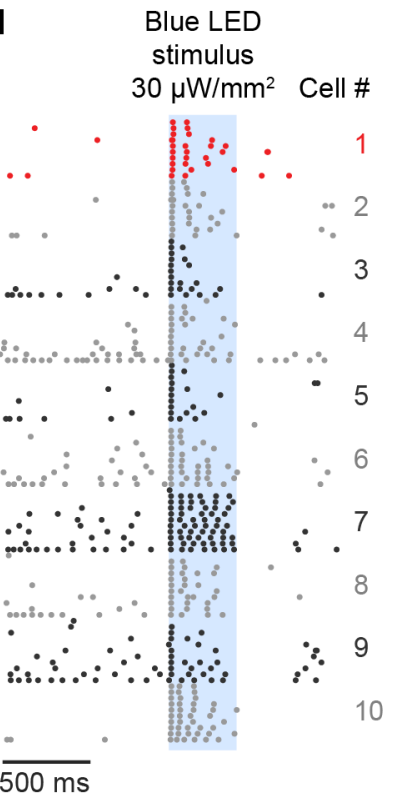

Figure 4: Simultaneous voltage imaging and optogenetic stimulation. a. (Top) Average intensity projection of $457 \mathrm{~Hz}$ confocal images showing Voltron2 ${ }_{585}$-expressing cells labeled with $\mathrm{JF}_{585}$ in an acute slice of motor cortex. Pipette used for whole-cell recordings illustrated in red. (Bottom) Post-hoc confocal image showing pan-neuronal expression of ChR2-GFP in the same field of view (FOV) shown in top panel, with patched cell \#1 indicated by white arrow. b. Whole-cell membrane voltage (black traces) and corresponding Voltron2 fluorescent signal (red traces) from patched cell \#1 shown in a, showing responses to 400 ms stimulation with 10 (top), 30 (middle), and $50 \mu \mathrm{W} / \mathrm{mm}^{2}$ (bottom) blue light. c. Voltron2 585 signals (red and gray traces) recorded across 10 distinct cells in the FOV shown in a in response to $400 \mathrm{~ms}$ stimulation with $30 \mu \mathrm{W} / \mathrm{mm}^{2}$ blue light. Corresponding membrane voltage is shown for patched cell \# 1 (upper black trace). d. Raster plots show trialaligned APs detected in fluorescent signals from cells \#1-10 shown in a and c, across 10 repeated $400 \mathrm{~ms}$ blue stimulus trials. 
bioRxiv preprint doi: https://doi.org/10.1101/2021.11.09.467909; this version posted November 11,2021 . The copyright holder for this preprint (which was not certified by peer review) is the author/funder, who has granted bioRxiv a license to display the preprint in perpetuity. It is made available under aCC-BY-NC-ND 4.0 International license.
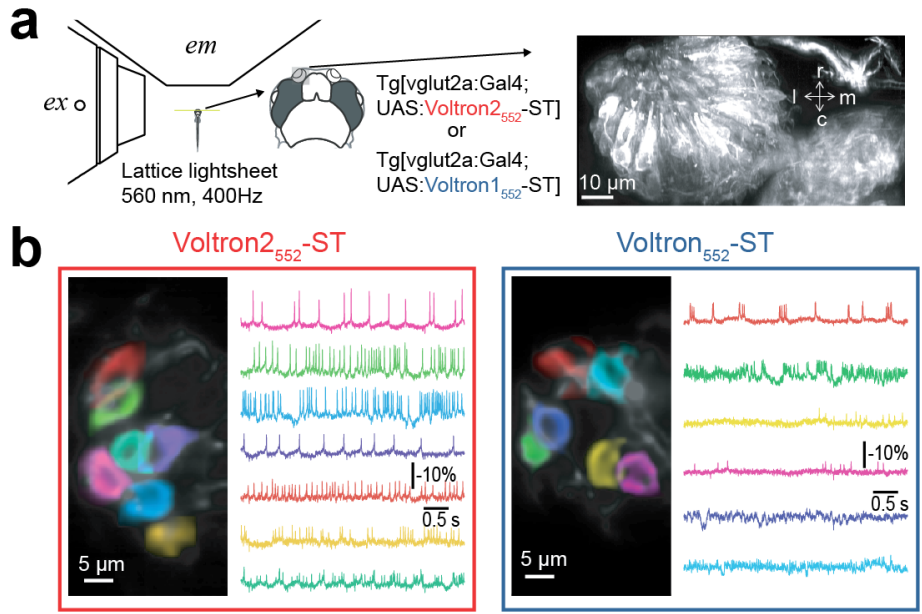

C
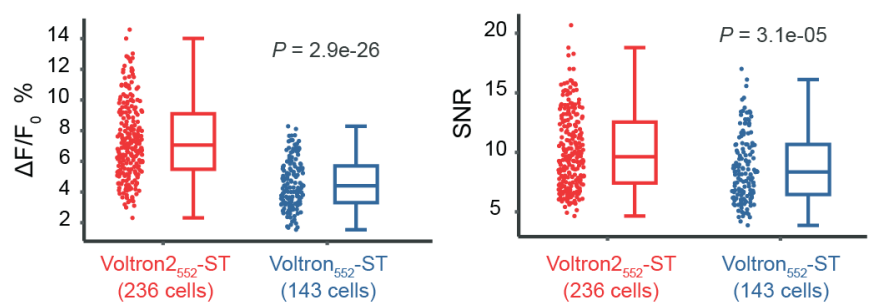

Figure 5: In vivo comparison of Voltron2-ST and Voltron-ST in zebrafish olfactory sensory neurons. a. Experimental setup. Left: Olfactory sensory neurons expressing Voltron2-ST or Voltron-ST, labeled with $\mathrm{JF}_{552}$ and imaged at $400 \mathrm{~Hz}$ using a lattice-lightsheet microscope. ex: excitation objective lens, em: imaging objective lens. Right: Volumetric rendering of olfactory sensory neurons in the nasal cavity. r, rostral; c, cadual; $\mathrm{m}$, medial; I, lateral b. Representative FOVs and recordings. Spatial weights optimized for individual spiking neurons are shown in distinct colors over the structural image (left). The activity trace of corresponding neurons is shown in the same color (right). c. Performance comparisons of Voltron2 ${ }_{552}-\mathrm{ST}$ and Voltron ${ }_{552}-\mathrm{ST}$. Left: Distribution of spike-related fluorescence change of Voltron2 ${ }_{552}-\mathrm{ST}$ and Voltron ${ }_{552}-\mathrm{ST}$. Right: Distribution of SNR of Voltron2 ${ }_{552}-\mathrm{ST}$ and Voltron ${ }_{552}$-ST. Statistical differences were assessed with the Wilcoxon rank-sum test. 
bioRxiv preprint doi: https://doi.org/10.1101/2021.11.09.467909; this version posted November 11,2021 . The copyright holder for this preprint (which was not certified by peer review) is the author/funder, who has granted bioRxiv a license to display the preprint in perpetuity. It is made available under aCC-BY-NC-ND 4.0 International license.
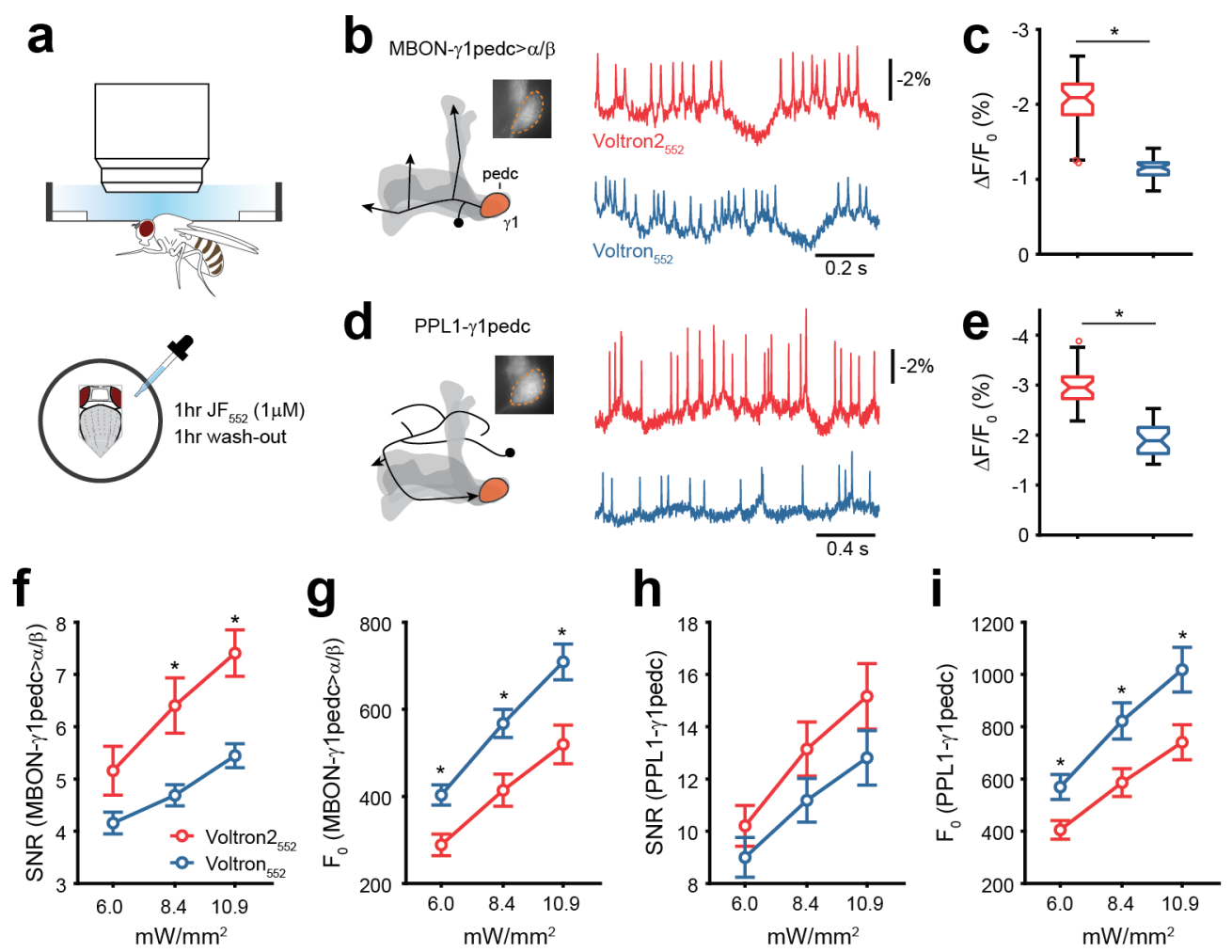

Figure 6: a. Experimental setup. A head-fixed fly is imaged using an sCMOS camera at 800 frames per second. Voltron is loaded with $\mathrm{JF}_{552}-\mathrm{Halotag}$ ligand via a $1 \mathrm{hr}$ incubation/1hr wash-out protocol. b,d. Voltage recordings in MBON- $\gamma 1$ pedc> $\alpha / \beta$ (MB112C-Gal4) and PPL1- $/ 1$ pedc (MB320C-Gal4). Neuron schematics are shown for the left hemisphere with the MB in shaded gray (arrowheads indicate axonal outputs). Fluorescence images were acquired from the $\gamma 1$ compartment (inset, $50 \mu \mathrm{m} \times 50 \mu \mathrm{m}$ ), which contains dendrites of MBON$\gamma 1$ pedc $>\alpha / \beta$ and axon terminals of PPL1- $\gamma 1$ pedc. Single-trial recordings of $\Delta F / F$ traces are shown $(8.4$ and 6.0 $\mathrm{mW} / \mathrm{mm}^{2}$ for $b$ and $d$ respectively). c. Spike amplitude with Voltron2 ${ }_{552}$ and Voltron 552 in MBON- $\gamma 1$ pedc $>\alpha / \beta$. P $<0.001$, Wilcoxon rank sum test. For Voltron2 552 , the data set was from 15 hemispheres (8 flies) at three levels of illumination for a total of 45 experiments, for Voltron ${ }_{552}, 13$ hemispheres ( 7 flies) with 39 experiments. e. Spike amplitude in PPL1-y1pedc. $\mathrm{P}<0.001$, Wilcoxon rank sum test. For both Voltron2 ${ }_{552}$ and Voltron 552 , the dataset was from 10 flies at three levels of illumination for 30 total experiments. $f, h$. SNR calculated as spike amplitude over standard deviation of the spike-free zones of the trace. $P=0.07,0.006,0.003$ between Voltron2 ${ }_{552}$ and Voltron $_{552}$ in MBON-y1pedc $>\alpha / \beta, P=0.28,0.16,0.17$ in PPL1- $\gamma 1$ pedc, two-sample t-test. g,i. Lower basal florescence levels with Voltron2552. $P<0.01$ in MBON- 1 1pedc $>\alpha / \beta, P<0.05$ in PPL1- $\gamma 1$ pedc, two-sample t-test. * indicates $\mathrm{P}<0.05$. 


\section{in vivo mouse hippocampus (PV neurons)}
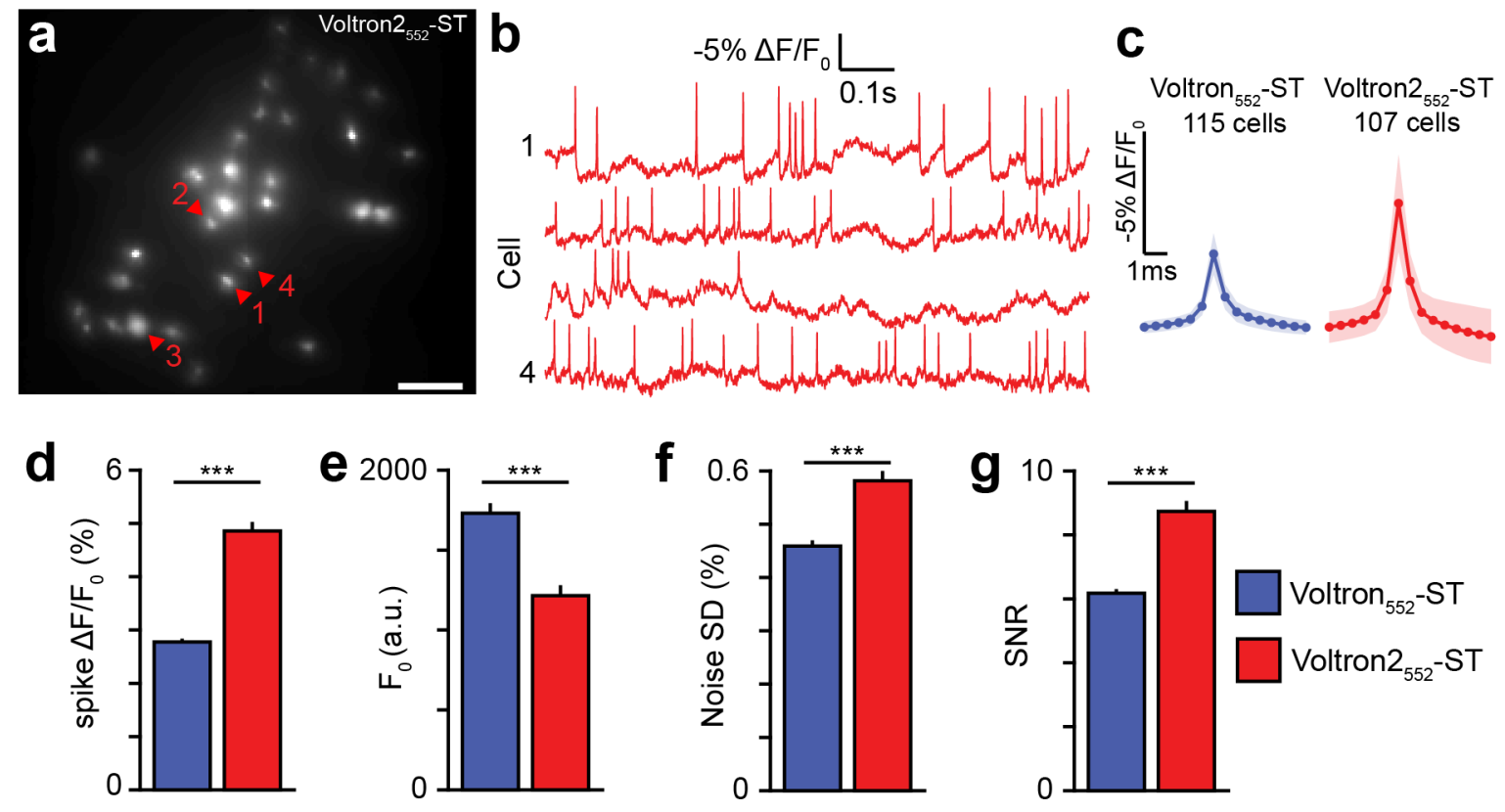

\section{in vivo mouse cortex}
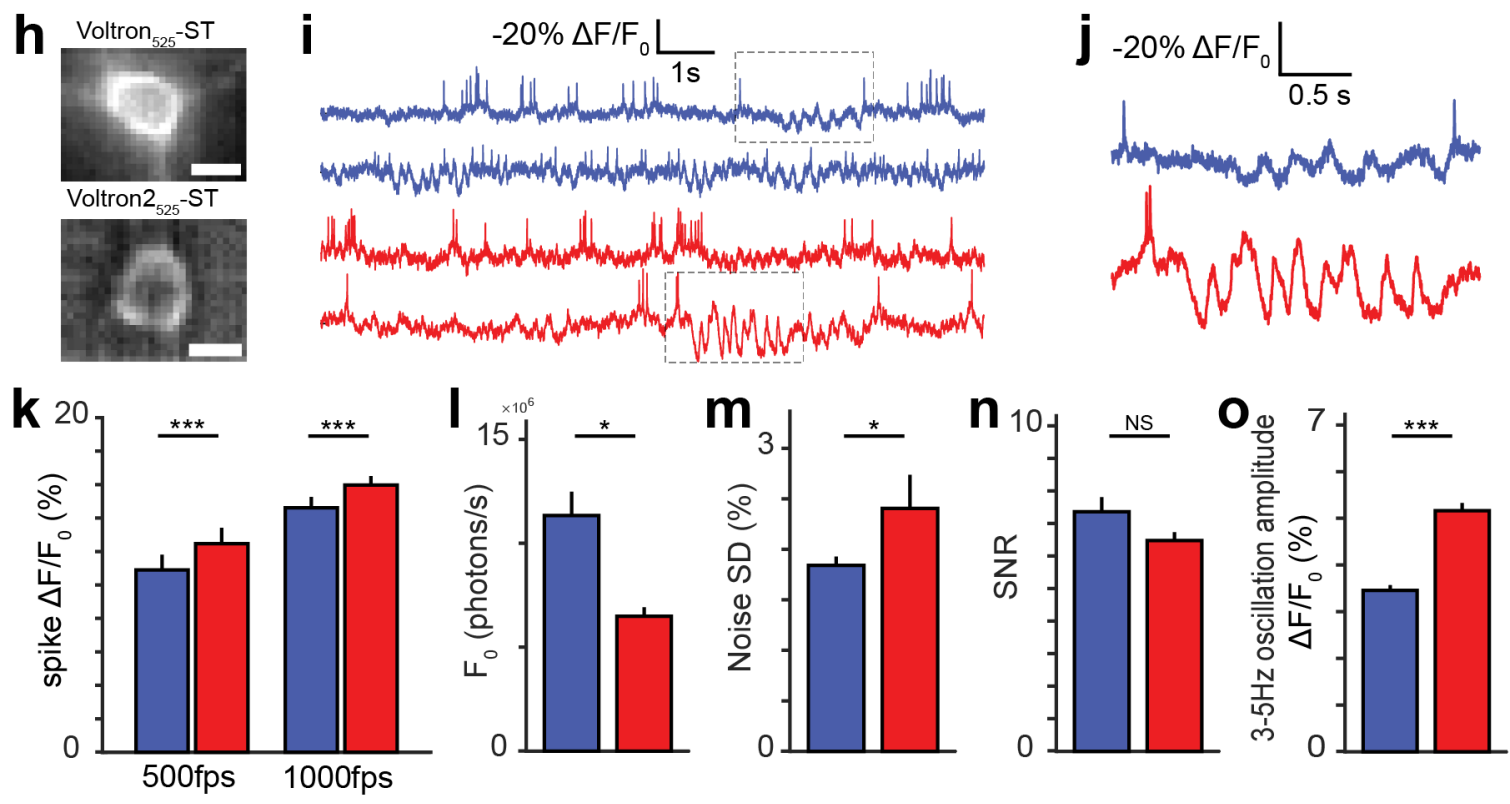

Figure 7: Imaging of voltage activity in vivo in mouse hippocampus and cortex with Voltron and Voltron2. a. Example image of hippocampal PV neurons expressing Voltron2-ST labeled with $\mathrm{JF}_{552 .}$. b. Sample fluorescence traces of cell 1 to 4 in (a). c. Average spike waveforms of cells expressing Voltron ${ }_{552}-S T$ or Voltron2 ${ }_{552}$-ST. d-g. Comparison of Voltron ${ }_{552}-\mathrm{ST}$ and Voltron2 ${ }_{552}$-ST spike amplitude (d), baseline fluorescence (e), noise standard deviation (f), and SNR (g) in hippocampal PV neurons. h. Example images of

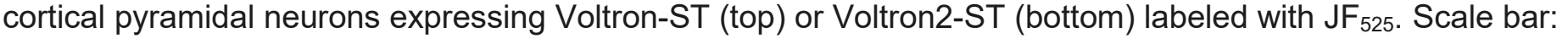
$10 \mu \mathrm{m}$. i. Example fluorescence traces from individual neurons recorded using Voltron ${ }_{525}-\mathrm{ST}$ (blue) and Voltron ${ }_{525}$-ST (red) detrended using a 5 s median filter. Grey dashed boxes indicate detection of 3-5Hz oscillations shown in (j) and quantified in (o). j. Zoomed portions of the fluorescence traces in (i) showing spikes and $3-5 \mathrm{~Hz}$ oscillations. k-0. Comparison of Voltron ${ }_{525}-\mathrm{ST}$ and Voltron2 ${ }_{525}$-ST spike amplitude at both 500 and 1000 frames per second imaging rates $(\mathrm{k})$, baseline fluorescence $(\mathrm{l})$, noise standard deviation $(\mathrm{m})$, SNR (n), and 3-5Hz oscillation amplitude (o) in cortical pyramidal neurons. For all plots: Statistically significant 
bioRxiv preprint doi: https://doi.org/10.1101/2021.11.09.467909; this version posted November 11,2021 . The copyright holder for this preprint (which was not certified by peer review) is the author/funder, who has granted bioRxiv a license to display the preprint in perpetuity. It is made available under aCC-BY-NC-ND 4.0 International license.

differences between groups were determined by Wilcoxon rank-sum test. ${ }^{*} p<0.05,{ }^{* *} p<0.01,{ }^{* * *} p<0.001$. Error bars indicate SEM.
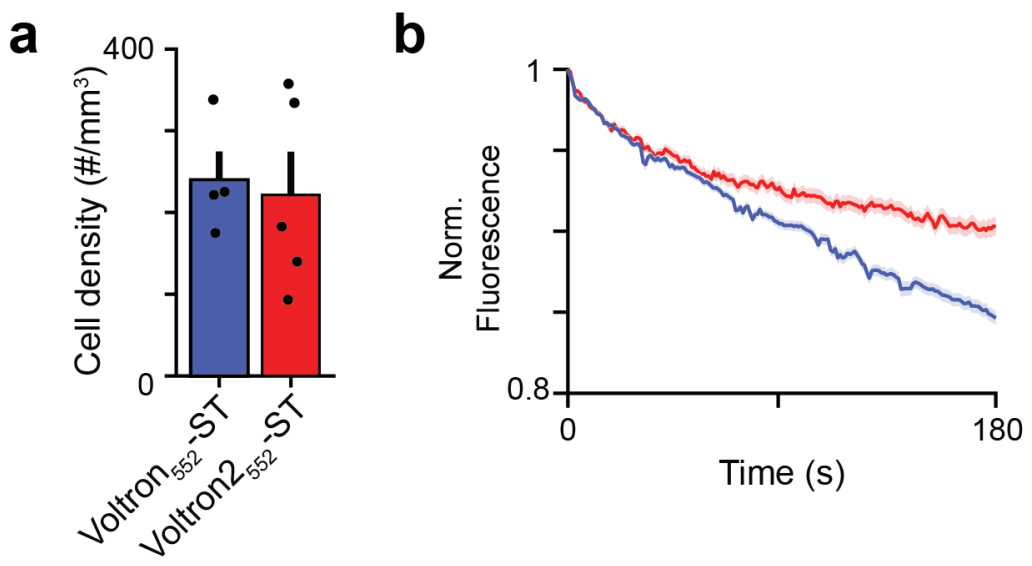

Supplementary Figure 11: a. Density of visually identifiable neurons in mouse CA1. b. Photobleaching comparison of Voltron 552 and Voltron2 ${ }_{552}$ in mouse CA1 (solid color: mean, shading: SEM). 
bioRxiv preprint doi: https://doi.org/10.1101/2021.11.09.467909; this version posted November 11, 2021. The copyright holder for this preprint (which was not certified by peer review) is the author/funder, who has granted bioRxiv a license to display the preprint in perpetuity. It is made available under aCC-BY-NC-ND 4.0 International license.

\section{Tables}

Supplementary Table 1: Screening results of field stimulation assay on Voltron point mutants. Normalization performed to in-plate Voltron controls.

Supplementary Table 2: Screening results of field stimulation assay on Voltron combo mutants. Normalization performed to in-plate Voltron controls.

Supplementary Table 3: Combo variants containing the A122D mutation, arranged by the number of mutations. Data aggregated from Supplementary Table 2.

Supplementary Table 4: Custom primers used for library tagmentation and NextSeq sequencing 